\title{
ON THE DIRECT PRODUCT OF BANACH SPACES
}

\author{
BY \\ ROBERT SCHATTEN
}

Introduction. Two Banach spaces $E_{1}$ and $E_{2}$ may be combined in two different ways; the well known $E_{1} \oplus E_{2}$ and $E_{1} \otimes E_{2}$. While $E_{1} \oplus E_{2}$ refers to a space of pairs $\{f, \varphi\}$ which are added vectorially, $E_{1} \otimes E_{2}$ is a linear vector space determined by "products" $f \otimes \varphi$, and the $f \otimes \varphi$ 's are linearly independent except when we have a relation, which is a consequence of the fact that the $\otimes$ operator is distributive; for instance

$$
\left(f_{1}+f_{2}\right) \otimes\left(\varphi_{1}+\varphi_{2}\right)=f_{1} \otimes \varphi_{1}+f_{1} \otimes \varphi_{2}+f_{2} \otimes \varphi_{1}+f_{2} \otimes \varphi_{2} .
$$

The notion of $E_{1} \otimes E_{2}$ for the case of finite dimensions has been already mentioned by $\mathrm{H}$. Weyl [16]( $\left.{ }^{1}\right)$. With each vector $e_{1}$ resp. $e_{2}$ in a space $E_{1}$ of $m$, resp. $E_{2}$ of $n$ dimensions, there is associated a vector $e_{1} \otimes e_{2}$ in the space of $m \cdot n$ dimensions. The totality of vectors $e_{1} \otimes e_{2}$ do not themselves constitute a linear manifold, but their linear combinations fill the entire "product space" $E_{1} \otimes E_{2}$.

The operator $\otimes$ has been used for finite-dimensional $l_{p}$ (termed $l_{p, n}$ ) by F. J. Murray, in order to show that there exist linear manifolds without complements [8]. It has also been used by the same author in treating bilinear transformations in Hilbert spaces [9].

The algebraic aspects of the $\otimes$ operator, for the case of finite-dimensional spaces, has been discussed by Hitchcock [5, 6], and Oldenburger [13, 14].

The study of the $\otimes$ operator for infinite-dimensional spaces requires a more abstract method. A complete discussion for Hilbert spaces has been given by F. J. Murray and J. von Neumann [10], who did not make use of the existence of a basis. A few special results for $L_{p}$ spaces have been obtained by Bourgin [2].

It should be pointed out, however, that so far the study of the $\otimes$ operator, assumed either the existence of an inner product, or a basis, or a projection with bound 1 .

The object of this paper is the study of the $\otimes$ operator in a most general form, for any Banach spaces.

For $f \in E_{1}, \varphi \in E_{2}$, we construct "products" $f \otimes \varphi$. With these we form a linear set $\mathfrak{A}\left(E_{1}, E_{2}\right)$ consisting of all "expressions" (that is, finite sums) $\sum f_{i} \otimes \varphi_{i}$. These expressions must first be considered algebraically, however, since the distributive property introduces certain linear dependencies $(\$ \S 1,2)$. The next problem is that of defining a norm, and the space $E_{1} \otimes E_{2}$ is obtained

Presented to the Society, October 25, 1941; received by the editors January 26, 1942.

(1) Numerals in brackets refer to bibliography at the end of this paper. 
by "closing up" the set $\mathfrak{A}\left(E_{1}, E_{2}\right)$. We are only interested in those norms which are "crossnorms" that is, $\|f \otimes \varphi\|=\|f\|\|\varphi\|$ for $f \in E_{1}, \varphi \in E_{2}$. With the expressions $\sum f_{i} \otimes \varphi_{i}$ it is desirable to consider the set $\mathfrak{A}\left(\bar{E}_{1}, \bar{E}_{2}\right)$ of expressions

$$
\sum F_{i} \otimes \phi_{i}, \quad \text { where } F_{i} \in \bar{E}_{1}, \phi_{i} \in \bar{E}_{2} .
$$

For a given norm in $\mathfrak{A}\left(E_{1}, E_{2}\right)$ we construct an associate norm in $\mathfrak{A}\left(\bar{E}_{1}, \bar{E}_{2}\right)$ (\$3). We prove the existence of the greatest crossnorm, the least crossnorm whose associate is also a crossnorm $(\$ 4)$, as well as that of a "self-associate" crossnorm, which is a generalization of the crossnorm given for Hilbert spaces by F. J. Murray and J. von Neumann [10], (\$5).

Finally, we mention a few unsolved problems in connection with the work in preceding sections $(\$ 6)$, and indicate possibilities for the construction of "general crossnorms" ( $\$ 7)$.

These problems were suggested to me by Professor F. J. Murray, who also pointed out the algebraic discussions given in $\$ \$ 1,2$.

1. Let $E_{1}$ and $E_{2}$ denote two linear spaces. We introduce two symbols $\otimes$ and $\cdot+\cdot$ With these for $f_{1}, \cdots, f_{n}$ in $E_{1}$ and $\varphi_{1}, \cdots, \varphi_{n}$ in $E_{2}$ we construct formal expressions $f_{1} \otimes \varphi_{1} \cdot+\cdot f_{2} \otimes \varphi_{2} \cdot+\cdots \cdot+\cdot f_{n} \otimes \varphi_{n}$. We may abbreviate this by writing $\sum_{i=1}^{n} f_{i} \otimes \varphi_{i}$. Between these we introduce a relation $\sim$ subject to the following rules:

1. If $P$ is a permutation on $1,2, \cdots, n$, and $P(i)$ denotes the integer into which $P$ takes $i$, then

$$
\sum_{i=1}^{n} f_{i} \otimes \varphi_{i} \sim \sum_{i=1}^{n} f_{P(i)} \otimes \varphi_{P(i)}
$$

2a. $\left(f_{1}^{\prime}+f_{1}^{\prime \prime}\right) \otimes \varphi_{1} \cdot+\cdot f_{2} \otimes \varphi_{2} \cdot+\cdot \cdots \cdot+\cdot f_{n} \otimes \varphi_{n} \sim f_{1}^{\prime} \otimes \varphi_{1} \cdot+\cdot f_{1}^{\prime \prime} \otimes \varphi_{1} \cdot+\cdot f_{2}$ $\otimes \varphi_{2}+\cdots \cdot+\cdot f_{n} \otimes \varphi_{n}$

2b. $f_{1} \otimes\left(\varphi_{1}^{\prime}+\varphi_{1}^{\prime \prime}\right) \cdot+\cdot f_{2} \otimes \varphi_{2} \cdot+\cdot \cdots+\cdot f_{n} \otimes \varphi_{n} \sim f_{1} \otimes \varphi_{1}^{\prime} \cdot+\cdot f_{1} \otimes \varphi_{1}^{\prime \prime} \cdot+\cdot f_{2}$ $\otimes \varphi_{2} \cdot+\cdots \cdot+f_{n} \otimes \varphi_{n}$.

3. $\left(a_{1} f_{1}\right) \otimes \varphi_{1} \cdot+\cdot\left(a_{2} f_{2}\right) \otimes \varphi_{2} \cdot+\cdot \cdot \cdot+\cdot\left(a_{n} f_{n}\right) \otimes \varphi_{n} \sim f_{1} \otimes\left(a_{1} \varphi_{1}\right) \cdot+\cdot f_{2} \otimes\left(a_{2} \varphi_{2}\right)$ $+\cdots \cdot \cdot \cdot f_{n} \otimes\left(a_{n} \varphi_{n}\right)$.

DeFinition 1.1. Two expressions $\sum_{i=1}^{n} f_{i} \otimes \varphi_{i}$ and $\sum_{j=1}^{m} g_{j} \otimes \psi_{j}$ will be termed equivalent, if one can be transformed into the other by a finite number of successive applications of Rules 1, 2, 3. We write this $\sum_{i=1}^{n} f_{i} \otimes \varphi_{i} \simeq \sum_{j=1}^{m} g_{j} \otimes \psi_{j}$.

Rule 1 shows that $\simeq$ is reflexive, that is, every expression is equivalent to itself. The definition also implies transitivity.

A number of elementary results can be easily obtained. For instance, if

$$
\sum_{i=1}^{n} f_{i} \otimes \varphi_{i} \simeq \sum_{i=1}^{n^{\prime}} f_{i}^{\prime} \otimes \varphi_{i}^{\prime} \quad \text { and } \quad \sum_{j=1}^{m} g_{j} \otimes \psi_{j} \simeq \sum_{j=1}^{m^{\prime}} g_{j}^{\prime} \otimes \psi_{i}^{\prime}
$$

then 


$$
\sum_{i=1}^{n} f_{i} \otimes \varphi_{i} \cdot+\cdot \sum_{j=1}^{m} g_{j} \otimes \psi_{j} \simeq \sum_{i=1}^{n^{\prime}} f_{i}^{\prime} \otimes \varphi_{i}^{\prime} \cdot+\cdot \sum_{j=1}^{m^{\prime}} g_{j}^{\prime} \otimes \psi_{j}^{\prime}
$$

Because of Rules $2 \mathrm{a}, 2 \mathrm{~b}, \boldsymbol{n}$ is not an invariant under equivalence for the expression $\sum_{i=1}^{n} f_{i} \otimes \varphi_{i}$. However, we now define a quantity "the rank of $\sum_{i=1}^{n} f_{i} \otimes \varphi_{i}$ " which will be shown to be an invariant.

Definition 1.2. Consider $\sum_{i=1}^{n} f_{i} \otimes \varphi_{i}$. Let us suppose that the set of $f_{i}$ 's is $k$-dimensional and that the set of $\varphi_{i}^{\prime}$ 's is l-dimensional. Let $g_{1}, \cdots, g_{k}$ be $k$ linearly independent elements in the set of linear combinations of the $f_{i}$, and $\psi_{1}, \cdots, \psi_{l}$ be $l$ linearly independent elements in the set of linear combinations of the $\varphi_{i}$. Then

and

$$
f_{i}=\sum_{p=1}^{k} a_{p}^{(i)} g_{p}, \quad \varphi_{i}=\sum_{q=1}^{l} b_{q}^{(i)} \psi_{q} \quad \text { for } 1 \leqq i \leqq n,
$$

$$
\begin{aligned}
\sum_{i=1}^{n} f_{i} \otimes \varphi_{i} & \simeq \sum_{i=1}^{n}\left(\sum_{p=1}^{k} a_{p}^{(i)} g_{p}\right) \otimes\left(\sum_{q=1}^{l} b_{q}^{(i)} \psi_{q}\right) \\
& \simeq \sum_{p=1}^{k} \sum_{q=1}^{l}\left(\sum_{i=1}^{n} a_{p}^{(i)} b_{q}^{(i)}\right) g_{p} \otimes \psi_{q} \\
& \simeq \sum_{p=1}^{k} \sum_{q=1}^{l} a_{p, q} g_{p} \otimes \psi_{q} .
\end{aligned}
$$

We define the "rank of the expression $\sum_{i=1}^{n} f_{i} \otimes \varphi_{i}$ " as the rank of the matrix $\left(a_{p, q}\right) p=1, \cdots, k ; q=1, \cdots, l$.

To justify this definition we show that the rank does not depend upon the choice of the $g_{p}$ or $\psi_{q}$. For suppose we had taken $g_{1}^{\prime}, \cdots, g_{k}^{\prime}$ and $\psi_{1}^{\prime}, \cdots, \psi_{l}^{\prime}$ instead of $g_{1}, \cdots, g_{k}$ and $\psi_{1}, \cdots, \psi_{l}$ above. Then we can show that

$$
\sum_{i=1}^{n} f_{i} \otimes \varphi_{i} \simeq \sum_{p=1}^{k} \sum_{q=1}^{l} a_{p, q}^{\prime} g_{p}^{\prime} \otimes \psi_{q}^{\prime}
$$

where $\left(a_{p, q}^{\prime}\right)$ is equal to a product $B\left(a_{p, q}\right) C ; B$ resp. $C$ being nonsingular square matrices with $k$ resp. $l$ rows. But the matrix $B\left(a_{p, q}\right) C$ is of the same rank as $\left(a_{p, q}\right)$. Thus the rank of an expression is independent of the choice of the $g_{p}$ and $\psi_{q}$.

Let us define $f_{q}^{*}$ by means of the equation $f_{q}^{*}=\sum_{p-1}^{k} a_{p, q} g_{p}$. Then, inasmuch as the $\psi_{j}$ are linearly independent, the rank of the expression $\sum_{i=1}^{n} f_{i} \otimes \varphi_{i}$ is the number of $f_{q}^{*}$ which are linearly independent.

LEMMA 1.1. The rank $r$ of an expression $\sum_{i=1}^{n} f_{i} \otimes \varphi_{i}$ is an invariant under equivalence (Definition 1.1). 
Proof. It is easily seen that Rule 1 does not affect $r$. In $2 \mathrm{a}$, let $f_{1}=f_{1}^{\prime}+f_{1}^{\prime \prime}$, $h=(1 / 2)\left(f_{1}^{\prime}-f_{1}^{\prime \prime}\right)$. Then $f_{1}^{\prime}=(1 / 2) f_{1}+h, f_{1}^{\prime \prime}=(1 / 2) f_{1}-h$,

$$
\begin{aligned}
f_{1} \otimes \varphi_{1} \cdot+\cdot \sum_{i=2}^{n} f_{i} \otimes \varphi_{i} \simeq & \left((1 / 2) f_{1}+h\right) \otimes \varphi_{1} \\
& +\cdot \cdot\left((1 / 2) f_{1}-h\right) \otimes \varphi_{1} \cdot+\cdot \sum_{i=2}^{n} f_{i} \otimes \varphi_{i} .
\end{aligned}
$$

Now, if $h=\sum_{p=1}^{k} a_{p} g_{p}$, a calculation shows that the $f_{q}^{*}$ in the above are the same for both sides of the relation. On the other hand, if $h$ is linearly independent of $g_{1}, \cdots, g_{k}$, we may put $h=g_{k+1}$. But $g_{k+1}$ will appear in the $f_{q}^{*}$ only with zero coefficients and again the $f_{q}^{*}$ are the same for both sides. Since $r$ is the number of linearly independent $f_{q}^{*}, r$ must be also the same on both sides.

A similar discussion will show that $r$ is also unaffected by $2 \mathrm{~b}$.

In 3 , if any term $a_{i}$ is zero, we may take $f_{i}$ as zero. If $\varphi_{i}$ is also zero, we may disregard the term. If $\varphi_{i}$ is not zero, it may be taken as $\psi_{1}$. Then it is easily seen that this term does not contribute to the $f_{q}^{*}$, and hence does not affect the rank. Thus an expression $\sum_{i=1}^{n}\left(a_{i} f_{i}\right) \otimes \varphi_{i}$ has the same rank as an expression in which the terms with $a_{i}=0$ are disregarded. A similar statement holds for $\sum_{i=1}^{n} f_{i} \otimes\left(a_{i} \varphi_{i}\right)$.

This implies that we need consider only the case in which each $a_{i} \neq 0$. But here we may take the same $g_{p}$ and $\psi_{q}$ on both sides of the relation. We then see that $a_{p}^{(i)} b_{q}^{(i)}$ has the same value for both sides. Hence the matrix $\left(a_{p, q}\right)$ and the rank are the same in both cases.

Thus the rank is unaffected by each of these rules. It follows that it is unaffected by any sequence of applications of these rules, and hence it is invariant under equivalence.

LEMMA 1.2. Every expression $\sum_{i=1}^{n} f_{i} \otimes \varphi_{i}$ is equivalent to either $0 \otimes 0$, or to an expression $\sum_{i=1}^{m} g_{i} \otimes \psi_{i}$ in which both the $g_{1}, \cdots, g_{m}$ and $\psi_{1}, \cdots, \psi_{m}$ are linearly independent. Furthermore, $m$ equals the rank of $\sum_{i=1}^{n} f_{i} \otimes \varphi_{i}$.

Proof. It is readily seen that if in either of the sets $f_{1}, \cdots, f_{n} ; \varphi_{1}, \cdots, \varphi_{n}$ the elements are linearly dependent, then $\sum_{i=1}^{n} f_{i} \otimes \varphi_{i}$ is equivalent to an expression involving only $n-1$ terms. For instance, if $f_{1}=\sum_{i=2}^{n} a_{i} f_{i}$, then

$$
\begin{aligned}
f_{1} \otimes \varphi_{1} \cdot+\cdot \sum_{i=2}^{n} f_{i} \otimes \varphi_{i} & \simeq\left(\sum_{i=2}^{n} a_{i} f_{i}\right) \otimes \varphi_{1} \cdot+\cdot \sum_{i=2}^{n} f_{i} \otimes \varphi_{i} \\
& \simeq \sum_{i=2}^{n}\left(a_{i} f_{i}\right) \otimes \varphi_{1} \cdot+\cdot \sum_{i=2}^{n} f_{i} \otimes \varphi_{i} \\
& \simeq \sum_{i=2}^{n} f_{i} \otimes\left(a_{i} \varphi_{1}\right)+\cdot \sum_{i=2}^{n} f_{i} \otimes \varphi_{i} \\
& \simeq \sum_{i=2}^{n} f_{i} \otimes\left(a_{i} \varphi_{1}+\varphi_{i}\right) .
\end{aligned}
$$


We may therefore continue to reduce the number of terms until we have either $\sum_{t=1}^{m} g_{i} \otimes \psi_{i}$, in which both the $g_{1}, \cdots, g_{m}$ and $\psi_{1}, \cdots, \psi_{m}$ are linearly independent or $f \otimes 0$, or $0 \otimes \varphi$. But $f \otimes 0 \simeq f \otimes(0 \cdot 0) \simeq(0 f) \otimes 0 \simeq 0 \otimes 0$. Similarly $0 \otimes \varphi \simeq 0 \otimes 0$.

Now the expression $\sum_{i=1}^{m} g_{i} \otimes \psi_{i}$ with both the $g_{i}$ and $\psi_{i}$ linearly independent has rank $m$. For the $g_{i}$ and $\psi_{i}$ can be used as in Definition 1.2. The resulting matrix is $\left(\delta_{i j}\right), i, j=1, \cdots, m$. Since, however, the rank of an expression is invariant under equivalence (Lemma 1.1), $m$ must be also the rank of $\sum_{i=1}^{n} f_{i} \otimes \varphi_{i}$.

LEMMA 1.3. Suppose that in the expression $\sum_{i=1}^{n} f_{i} \otimes \varphi_{i}$ the $\varphi_{i}$ 's are linearly independent. Then the rank of this expression is $r$, the number of the $f_{i}$ 's which are linearly independent. In particular, if $\sum_{i=1}^{n} f_{i} \otimes \varphi_{i} \simeq 0 \otimes 0$, each $f_{i}$ is zero.

Proof. If $r=0$, the rank is zero also. Suppose $r \neq 0$. We may assume that $f_{1}, \cdots, f_{r}$ are linearly independent, since otherwise a permutation of the terms (Rule 1) will give this. Then $f_{r+p}=\sum_{k=1}^{r} a_{p, k} f_{k}$. Hence

where

$$
\begin{aligned}
\sum_{k=1}^{n} f_{k} \otimes \varphi_{k} & \simeq \sum_{k=1}^{r} f_{k} \otimes \varphi_{k}+\cdot \sum_{p=1}^{n-r}\left(\sum_{k=1}^{r} a_{p, k} f_{k}\right) \otimes \varphi_{r+p} \\
& \simeq \sum_{k=1}^{r} f_{k} \otimes\left(\varphi_{k}+\sum_{p=1}^{n-r} a_{p, k} \varphi_{r+p}\right) \simeq \sum_{k=1}^{r} f_{k} \otimes \psi_{k}
\end{aligned}
$$

$$
\psi_{k}=\varphi_{k}+\sum_{p=1}^{n-r} a_{p, k} \varphi_{r+p}, \quad \quad k=1, \cdots, r .
$$

Since the $\varphi_{i}$ 's are linearly independent, that is also true for the $\psi_{i}$ 's. But the rank of $\sum_{k=1}^{r} f_{k} \otimes \psi_{k}$ is $r$ (Lemma 1.2), therefore the rank of $\sum_{i=1}^{n} f_{i} \otimes \varphi_{i}$ is alsor (Lemma 1.1).

Furthermore, from Lemma 1.2, follows that an expression is equivalent to $0 \otimes 0$ if and only if its rank is 0 . From the preceding we see that the rank is zero, if and only if each $f_{i}$ is zero. This implies the last statement of our lemma.

COROLlaRY. If $\sum_{i=1}^{n} f_{i} \otimes \varphi_{i} \simeq \sum_{i=1}^{n} g_{i} \otimes \varphi_{i}$ and the $\varphi_{i}$ are linearly independent, then $f_{i}=g_{i}$ for $i=1, \cdots, n$.

LEMMA 1.4. If $\sum_{i=1}^{n} f_{i} \otimes \varphi_{i} \simeq 0 \otimes 0$, and $k$ of the $f_{i}$ and $l$ of the $\varphi_{i}$ are linearly independent, then $k+l \leqq n$.

Proof. Suppose that the $\varphi_{1}, \cdots, \varphi_{l}$ are linearly independent and, $\varphi_{l+i}=\sum_{j=1}^{l} a_{i, j} \varphi_{j}$ for $j=1, \cdots, n-l$. Then

$$
\begin{aligned}
0 \otimes 0 \simeq \sum_{j=1}^{n} f_{j} \otimes \varphi_{j} & \simeq \sum_{j=1}^{l} f_{j} \otimes \varphi_{j}+\cdot \sum_{j=1}^{l}\left(\sum_{i=1}^{n-l} a_{i, j} f_{l+i}\right) \otimes \varphi_{j} \\
& \simeq \sum_{j=1}^{l}\left(f_{j}+\sum_{i=1}^{n-l} a_{i, j} f_{l+i}\right) \otimes \varphi_{j} .
\end{aligned}
$$


Thus Lemma 1.3 implies $f_{j}+\sum_{i=1}^{n-l} a_{i, j} f_{l+i}=0$ for $j=1, \cdots, l$. The $l$ relations between the $f_{i}$ are linearly independent, and hence there can be at most $n-l$ of the $f_{i}$ linearly independent. Thus $k \leqq n-l$.

LEMMA 1.5. If $\sum_{i=1}^{n} f_{i} \otimes \varphi_{i} \simeq \sum_{j=1}^{m} g_{j} \otimes \psi_{j}$ and each of the sets $f_{1}, \cdots, f_{n}$; $\varphi_{1}, \cdots, \varphi_{n} ; g_{1}, \cdots, g_{m} ; \psi_{1}, \cdots, \psi_{m}$, are linearly independent, then $n=m$, and there exists a square matrix $\left(a_{i, j}\right), i, j=1, \cdots, n$, with an inverse $\left(A_{i, j}\right)$, $i, j=1, \cdots, n$, such that

$$
\psi_{k}=\sum_{j=1}^{n} a_{k, j} \varphi_{j} ; \quad g_{k}=\sum_{j=1}^{n} A_{j, k} f_{j} .
$$

Proof. Lemma 1.3 states that $m$ and $n$ are the ranks of the corresponding expressions. Thus Lemma 1.1 implies that they are equal.

Now $\sum_{i=1}^{n} f_{i} \otimes \varphi_{i} \simeq \sum_{j=1}^{n} g_{j} \otimes \psi_{j}$ implies

$$
\sum_{i=1}^{n} f_{i} \otimes \varphi_{i} \cdot+\cdot \sum_{j=1}^{n}\left(-g_{j}\right) \otimes \psi_{j} \simeq 0 \otimes 0 .
$$

Inasmuch as $f_{1}, \cdots, f_{n}$ are linearly independent, Lemma 1.4 implies that at most $n$ of the set $\varphi_{1}, \cdots, \varphi_{n}, \psi_{1}, \cdots, \psi_{n}$, are linearly independent. Since $\varphi_{1}, \cdots, \varphi_{n}$ are linearly independent, each $\psi_{k}$ must depend upon these. Thus $\psi_{k}=\sum_{j=1}^{n} a_{k, j} \varphi_{j}$. But since $\psi_{1}, \cdots, \psi_{n}$ are linearly independent, the matrix $\left(a_{i, j}\right), i, j=1, \cdots, n$ must have an inverse $\left(A_{i, j}\right), i, j=1, \cdots, n$, and $\varphi_{j}=\sum_{k=1}^{n} A_{j, k} \psi_{k}$. Substituting in (1), we get

$$
\sum_{k=1}^{n}\left(\sum_{i=1}^{n} A_{i, k} f_{i}-g_{k}\right) \otimes \psi_{k} \simeq 0 \otimes 0 .
$$

Lemma 1.3 now implies that $g_{k}=\sum_{i=1}^{n} A_{i, k} f_{i}$.

DEFINITION 1.3. The set of all expressions of the form $\sum_{i=1}^{n} f_{i} \otimes \varphi_{i}$, we denote by $\mathfrak{A}\left(E_{1}, E_{2}\right)$. Let $\bar{f}$ denote the set of all expressions equivalent to a fixed expression $\sum_{i-1}^{n} f_{i} \otimes \varphi_{i}$. If an expression is in $\bar{f}$ it will be termed "an expression for $\bar{f}$." Let $\mathfrak{Q}^{*}\left(E_{1}, E_{2}\right)$ denote the set of such $\bar{f}^{\prime} s$.

If $\sum_{i=1}^{n} f_{i} \otimes \varphi_{i}$ is an expression for $\bar{f}, \sum_{j=1}^{m} g_{j} \otimes \psi_{j}$ an expression for $\bar{g}, \alpha$ a number, we define $\alpha \bar{f}$ as the set of expressions equivalent to $\sum_{i=1}^{n}\left(\alpha f_{i}\right) \otimes \varphi_{i}$, and $\bar{f}+\bar{g}$ as the set of expressions equivalent to $\sum_{i=1}^{n} f_{i} \otimes \varphi_{i}+\cdot \cdot \sum_{j=1}^{m} g_{j} \otimes \psi_{j}$.

It is a consequence of Definition 1.1 and Rules $1,2,3$, that $\alpha \bar{f}$ does not depend on the particular expression used. Similarly $\bar{f}+\bar{g}$ is defined uniquely.

It is easy to see that the usual properties of addition and multiplication by a scalar hold; for instance $\bar{f}+\bar{g}=\bar{g}+\bar{f}$,

and

$$
\bar{f}+(\bar{g}+\bar{h})=(\bar{f}+\bar{g})+\bar{h}, \quad \alpha(\bar{f}+\bar{g})=\alpha \bar{f}+\alpha \bar{g}
$$

$$
(\alpha \beta) \bar{f}=\alpha(\beta \bar{f}) .
$$


The zero element $\overline{0}$ is the class of all expressions equivalent to $0 \otimes 0$. Thus $\mathfrak{Q}^{*}\left(E_{1}, E_{2}\right)$ is a linear set, that is, a commutative group with scalar operators.

Sometimes we will find it convenient to permit "an expression for $\bar{f}$ " to stand for $\bar{f}$.

2. LEMMA 2.1.a. If $F$ is an additive and homogenous functional on $E_{1}$, and $\sum_{i=1}^{n} f_{i} \otimes \varphi_{i} \simeq \sum_{j-1}^{m} g_{j} \otimes \psi_{j}$ then

$$
\sum_{i=1}^{n} F\left(f_{i}\right) \varphi_{i}=\sum_{j=1}^{m} F\left(g_{j}\right) \psi_{j} .
$$

Proof. Consider first $\sum_{i=1}^{n} f_{i} \otimes \varphi_{i}$. Suppose that $\varphi_{n}$ is linearly dependent on $\varphi_{1}, \cdots, \varphi_{n-1} ; \varphi_{n}=\sum_{i=1}^{n-1} a_{i} \varphi_{i}$. Then $\sum_{i=1}^{n} f_{i} \otimes \varphi_{i} \simeq \sum_{i=1}^{n-1}\left(f_{i}+a_{i} f_{n}\right) \otimes \varphi_{i}$ and

$$
\begin{aligned}
\sum_{i=1}^{n} F\left(f_{i}\right) \varphi_{i} & =\sum_{i=1}^{n-1} F\left(f_{i}\right) \varphi_{i}+F\left(f_{n}\right)\left(\sum_{i=1}^{n-1} a_{i} \varphi_{i}\right) \\
& =\sum_{i=1}^{n-1}\left(F\left(f_{i}\right)+a_{i} F\left(f_{n}\right)\right) \varphi_{i} \\
& =\sum_{i=1}^{n-1} F\left(f_{i}+a_{i} f_{n}\right) \varphi_{i} .
\end{aligned}
$$

A similar statement holds in the case in which $f_{n}$ is linearly dependent on $f_{1}, \cdots, f_{n-1}$. These results can be applied successively in such a way that one has finally $\sum_{i=1}^{n} f_{i} \otimes \varphi_{i} \simeq \sum_{i=1}^{k} f_{i}^{\prime} \otimes \varphi_{i}^{\prime}$ and $\sum_{i=1}^{n} F\left(f_{i}\right) \varphi_{i}=\sum_{i=1}^{k} F\left(f_{i}^{\prime}\right) \varphi_{i}^{\prime}$ with both the $f_{i}^{\prime}$ 's and $\varphi_{i}^{\prime}$ 's linearly independent. Suppose one has gone through the corresponding process with $\sum_{j-1}^{m} g_{j} \otimes \psi_{j}$. The conclusion of our lemma is then a simple consequence of Lemma 1.5.

Let $E_{1}^{\dagger}$ resp. $E_{2}^{\dagger}$ denote the set of additive and homogenous functionals on $E_{1}$ resp. $E_{2}$. For $F_{1}, \cdots, F_{n}$ in $E_{1}^{\dagger}$, and $\phi_{1}, \cdots, \phi_{n}$ in $E_{2}^{\dagger}$, we construct expressions $\sum_{i=1}^{n} F_{i} \otimes \phi_{i}$.

A similar reasoning to that in Lemma 2.1.a proves

LeMmA 2.1.b. If $\sum_{i=1}^{n} F_{i} \otimes \phi_{i} \simeq \sum_{j=1}^{m} G_{j} \otimes \Psi_{j}$ then for $f \subset E_{1}$, we have $\sum_{i=1}^{n} F_{i}(f) \phi_{i}=\sum_{j=1}^{m} G_{j}(f) \Psi_{j}$.

Combining these results we easily obtain

LEMMA 2.1.c. If

$$
\sum_{i=1}^{n} f_{i} \otimes \varphi_{i} \simeq \sum_{i=1}^{n^{\prime}} f_{i}^{\prime} \otimes \varphi_{i}^{\prime}
$$

and

then

$$
\sum_{j=1}^{m} F_{j} \otimes \phi_{j} \simeq \sum_{j=1}^{m^{\prime}} F_{i}^{\prime} \otimes \phi_{j}^{\prime}
$$




$$
\left(\sum_{j=1}^{m} F_{j} \otimes \phi_{j}\right)\left(\sum_{i=1}^{n} f_{i} \otimes \varphi_{i}\right)=\left(\sum_{j=1}^{m^{\prime}} F_{j}^{\prime} \otimes \phi_{j}^{\prime}\right)\left(\sum_{i=1}^{n^{\prime}} f_{i}^{\prime} \otimes \varphi_{i}^{\prime}\right)
$$

where under $\left(\sum_{j-1}^{m} F_{j} \otimes \phi_{j}\right)\left(\sum_{i=1}^{n} f_{i} \otimes \varphi_{i}\right)$ we understand

$$
\sum_{j=1}^{m} \sum_{i=1}^{n} F_{j}\left(f_{i}\right) \phi_{j}\left(\varphi_{i}\right)
$$

Definition 2.1. A set $S$ of additive and homogenous functionals on $E_{1}$, will be called fundamental if $F(f)=0$ for all $F \in S$, implies $f=0$.

Lemma 2.2. Let $S^{0}$ denote a fundamental set contained in $E_{1}^{\dagger}$, and $S^{00} a$ fundamental set contained in $E_{2}^{\dagger}$. Then if for an expression $\sum_{i=1}^{n} f_{i} \otimes \varphi_{i}$, we have $\sum_{i=1}^{n} F\left(f_{i}\right) \phi\left(\varphi_{i}\right)=0$ for all $F \in S^{0}, \phi \in S^{00}$ then $\sum_{i=1}^{n^{\prime}} f_{i} \otimes \varphi_{i} \simeq 0 \otimes 0$.

Proof. Suppose $\sum_{i=1}^{n} f_{i} \otimes \varphi_{i} \simeq \sum_{i=1}^{n^{\prime}} f_{i}^{\prime} \otimes \varphi_{i}^{\prime}$ where the $f_{i}^{\prime}$ 's and $\varphi_{i}^{\prime}$ 's are linearly independent, then for a certain $F^{0} \in S^{0}$ we have $F^{0}\left(f_{1}^{\prime}\right) \neq 0$, and since the $\varphi_{i}^{\prime}$ 's are linearly independent $\sum_{i=1}^{n^{\prime}} F^{0}\left(f_{i}^{\prime}\right) \varphi_{i}^{\prime} \neq 0$. Therefore for a certain $\phi^{0} \in S^{00}$ we have $\sum_{i=1}^{n^{\prime}} F^{0}\left(f_{i}^{\prime}\right) \phi^{0}\left(\varphi_{i}^{\prime}\right) \neq 0$. Lemma 2.1 gives $\sum_{i=1}^{n} F^{0}\left(f_{i}\right) \phi^{0}\left(\varphi_{i}\right) \neq 0$. This completes the proof.

TheOREm 2.1. Let $S^{0}$ resp. $S^{00}$ denote fundamental sets of additive and homogenous functionals on $E_{1}$ resp. $E_{2}$. Then, a necessary and sufficient condition, for the expressions

$$
\sum_{i=1}^{n} f_{i} \otimes \varphi_{i} \quad \text { and } \quad \sum_{i=1}^{n^{\prime}} f_{i}^{\prime} \otimes \varphi_{i}^{\prime}
$$

to be equivalent is that

$$
\sum_{i=1}^{n} F\left(f_{i}\right) \phi\left(\varphi_{i}\right)=\sum_{i=1}^{n^{\prime}} F\left(f_{i}^{\prime}\right) \phi\left(\varphi_{i}^{\prime}\right) \quad \text { for all } F \in S^{0}, \phi \in S^{00} .
$$

Proof. The necessity follows from Lemma 2.1. For the sufficiency put $f_{i}^{\prime}=-f_{n+i}, \varphi_{i}^{\prime}=\varphi_{n+i} ; 1 \leqq i \leqq n^{\prime}$, then according to our assumption,

$$
\sum_{i=1}^{n+n^{\prime}} F\left(f_{i}\right) \phi\left(\varphi_{i}\right)=0 \quad \text { for } F \in S^{0}, \phi \in S^{00} .
$$

Lemma 2.2 gives

$$
\sum_{i=1}^{n+n^{\prime}} f_{i} \otimes \varphi_{i} \simeq 0 \otimes 0 \quad \text { or } \quad \sum_{i=1}^{n} f_{i} \otimes \varphi_{i} \simeq \sum_{i=1}^{n^{\prime}} f_{i}^{\prime} \otimes \varphi_{i}^{\prime}
$$

3. For our further considerations we shall assume that $E_{1}$ and $E_{2}$ are two Banach spaces, and denote by $\bar{E}_{1}$ resp. $\bar{E}_{2}$ the space of linear functionals on $E_{1}$ resp. $E_{2}$.

Definition 3.1. Under a norm $N$ in $\mathfrak{A}\left(E_{1}, E_{2}\right)$ (Definition 1.3) we shall 
understand a non-negative function of expressions satisfying the following conditions:

I. $N\left(\sum_{i=1}^{n} f_{i} \otimes \varphi_{i}\right)=0$ if and only if $\sum_{i=1}^{n} f_{i} \otimes \varphi_{i} \simeq 0 \otimes 0$.

II. $N\left(\sum_{i=1}^{n}\left(a f_{i}\right) \otimes \varphi_{i}\right)=|a| N\left(\sum_{i=1}^{n} f_{i} \otimes \varphi_{i}\right)$ for any real number $a$.

III. $N\left(\sum_{i=1}^{n} f_{i} \otimes \varphi_{i} \cdot+\cdot \sum_{i=1}^{n^{\prime}} f_{i}^{\prime} \otimes \varphi_{i}^{\prime}\right) \leqq N\left(\sum_{i=1}^{n} f_{i} \otimes \varphi_{i}\right)+N\left(\sum_{i=1}^{n^{\prime}} f_{i}^{\prime} \otimes \varphi_{i}^{\prime}\right)$.

IV. $N\left(\sum_{i=1}^{n} f_{i} \otimes \varphi_{i}\right)=N\left(\sum_{i=1}^{n^{\prime}} f_{i}^{\prime} \otimes \varphi_{i}^{\prime}\right)$ if $\sum_{i=1}^{n} f_{i} \otimes \varphi_{i} \simeq \sum_{i=1}^{n^{\prime}} f_{i}^{\prime} \otimes \varphi_{i}^{\prime}$.

Definition 3.2. Consider the set $\mathfrak{A}\left(\bar{E}_{1}, \bar{E}_{2}\right)$ of expressions of the form $\sum_{j=1}^{m} F_{j} \otimes \phi_{j}$, where $F_{1}, \cdots, F_{m}$ are in $\bar{E}_{1}$, and $\phi_{1}, \cdots, \phi_{m}$ are in $\bar{E}_{2}$. For $a$ fixed expression $\sum_{j=1}^{m_{0}} F_{j}^{0} \otimes \phi_{j}^{0}$ in $\mathfrak{A}\left(\bar{E}_{1}, \bar{E}_{2}\right)$ we define $\bar{N}\left(\sum_{j=1}^{m_{0}} F_{j}^{0} \otimes \phi_{j}^{0}\right)$ as the smallest number satisfying the inequality

$$
\left|\left(\sum_{j=1}^{m_{0}} F_{j}^{0} \otimes \phi_{j}^{0}\right)\left(\sum_{i=1}^{n} f_{i} \otimes \varphi_{i}\right)\right| \leqq \bar{N}\left(\sum_{j=1}^{m_{0}} F_{j}^{0} \otimes \phi_{j}^{0}\right) N\left(\sum_{i=1}^{n} f_{i} \otimes \varphi_{i}\right)
$$

for all $\sum_{1=1}^{n} f_{i} \otimes \varphi_{i}$ in $\mathfrak{A}\left(E_{1}, E_{2}\right)$.

Thus $\bar{N}$ is a function of expressions in $\mathfrak{A}\left(\bar{E}_{1}, \bar{E}_{2}\right)$.

Lemma 3.1. If $\bar{N}$ is finite for every expression in $\mathfrak{A}\left(\bar{E}_{1}, \bar{E}_{2}\right)$, then $\bar{N}$ also satisfies conditions I-IV.

Proof. I. If $\sum_{j=1}^{m} F_{j} \otimes \phi_{j} \simeq 0 \otimes 0$, then Lemma 2.1.c gives $\bar{N}\left(\sum_{j=1}^{m} F_{j} \otimes \phi_{j}\right)=0$. Suppose $\sum_{j=1}^{m} F_{j} \otimes \phi_{j} \simeq \sum_{j=1}^{m^{\prime}} F_{j}^{\prime} \otimes \phi_{j}^{\prime}$ where the $F_{j}^{\prime}$ 's and $\phi_{j}^{\prime}$ 's are linearly independent (Lemma 2.2). If $f$ in $E_{1}$ is such that $F_{1}^{\prime}(f) \neq 0$ then $\sum_{j-1}^{m_{1}^{\prime}} F_{j}^{\prime}(f) \phi_{j}^{\prime} \neq 0$ since the $\phi_{j}^{\prime}$ 's are linearly independent. Let $\varphi \in E_{2}$ be such that $\sum_{j=1}^{m^{\prime}} F_{j}^{\prime}(f) \phi_{j}^{\prime}(\varphi) \neq 0$. Lemma 2.1.c gives $\left(\sum_{j=1}^{m} F_{j} \otimes \phi_{j}\right)(f \otimes \varphi) \neq 0$. This implies $\bar{N}\left(\sum_{j=1}^{m} F_{j} \otimes \phi_{j}\right)>0$.

II and III are immediate.

IV is a consequence of Lemma 2.1.c.

For any Banach space we may assume $E \subset \overline{\bar{E}}$.

Lemma 3.2. $\overline{\bar{N}} \leqq N$ for all expressions in $\mathfrak{A}\left(E_{1}, E_{2}\right) \subset \mathfrak{A}\left(\overline{\bar{E}}_{1}, \overline{\bar{E}}_{2}\right)$.

Proof. Let $f_{1}, f_{2}, \cdots$ resp. $\varphi_{1}, \varphi_{2}, \cdots$ denote elements in $E_{1}$ resp. $E_{2}$, $F_{1}, F_{2}, \cdots$ resp. $\phi_{1}, \phi_{2}, \cdots$, denote elements in $\bar{E}_{1}$ resp. $\bar{E}_{2} ; F_{1}^{\ddagger}, F_{2}^{\ddagger}, \cdots$ resp. $\phi_{1}^{\ddagger}, \phi_{2}^{\ddagger}, \cdots$ denote elements in $\overline{\bar{E}}_{1}$ resp. $\overline{\bar{E}}_{2}$. For an element $f^{0}$ in $E_{1}$, $F\left(f^{0}\right)=F^{\ddagger 0}(F)$ is an element of $\overline{\bar{E}}_{1}$, and $\left\|F^{\ddagger}\right\|=\left\|f^{0}\right\|$. Therefore an expression $\sum_{i=1}^{k} f_{i}^{0} \otimes \varphi_{i}^{0}$ will correspond to an expression $\sum_{i=1}^{k} F_{i}^{\ddagger 0} \otimes \phi_{i}^{\ddagger 0}$, where the $f_{i}^{0}$ and $F_{i}^{\ddagger 0}$, as well as the $\varphi_{i}^{0}$ and $\phi_{i}^{\ddagger 0}$ are connected by the above mentioned relation. We have

$$
\begin{aligned}
\overline{\bar{N}}\left(\sum_{i=1}^{k} f_{i}^{0} \otimes \varphi_{i}^{0}\right) & =\overline{\bar{N}}\left(\sum_{i=1}^{k} F_{i}^{\ddagger 0} \otimes \phi_{i}^{\ddagger 0}\right) \\
& =\sup \left(\left|\left(\sum_{i=1}^{k} F_{i}^{\ddagger 0} \otimes \phi_{i}^{\ddagger 0}\right)\left(\sum_{j=1}^{n} F_{j} \otimes \phi_{j}\right)\right| / \bar{N}\left(\sum_{j=1}^{n} F_{j} \otimes \phi_{j}\right)\right)
\end{aligned}
$$


where sup (that is, the least upper bound) is taken over all expressions $\sum_{j=1} F_{j} \otimes \phi_{j}$ in $\mathfrak{A}\left(\bar{E}_{1}, \bar{E}_{2}\right)$, which are not equivalent to $0 \otimes 0$ (of rank 0 ). Therefore

$$
\begin{aligned}
& \overline{\bar{N}}\left(\sum_{i=1}^{k} f_{i}^{0} \otimes \phi_{i}^{0}\right) \\
& \quad=\sup \left(\left|\left(\sum_{j=1}^{n} F_{j} \otimes \phi_{j}\right)\left(\sum_{i=1}^{k} \tilde{f}_{i}^{0} \otimes \hat{\varphi}_{i}^{0}\right)\right| / \bar{N}\left(\sum_{j=1}^{n} F_{j} \otimes \phi_{j}\right)\right) .
\end{aligned}
$$

Let $\epsilon>0$ be given. There exists an expression $\sum_{j=1}^{n_{0}} F_{j}^{0} \otimes \phi_{j}^{0}$ such that

$$
\begin{aligned}
& \left|\left(\sum_{j=1}^{n_{0}} F_{j}^{0} \otimes \phi_{j}^{0}\right)\left(\sum_{i=1}^{k} f_{i}^{0} \otimes \varphi_{i}^{0}\right)\right| / \bar{N}\left(\sum_{j=1}^{n_{0}} F_{j}^{0} \otimes \phi_{j}^{0}\right) \\
& \geqq \overline{\bar{N}}\left(\sum_{i=1}^{k} f_{i}^{0} \otimes \stackrel{\varphi}{0}_{i}\right)-\epsilon .
\end{aligned}
$$

From the definition of $\bar{N}$ for a given $N$, we have

$$
\left|\left(\sum_{j=1}^{n_{0}} F_{j}^{0} \otimes \phi_{i}^{0}\right)\left(\sum_{i=1}^{k} f_{i}^{0} \otimes \varphi_{i}^{0}\right)\right| / N\left(\sum_{i=1}^{k} f_{i}^{0} \otimes \varphi_{i}^{0}\right) \leqq \bar{N}\left(\sum_{j=1}^{n_{0}} F_{j}^{0} \otimes \phi_{i}^{0}\right) .
$$

The last two inequalities give

$$
N\left(\sum_{i=1}^{k} f_{i}^{0} \otimes \varphi_{i}^{0}\right) \geqq \overline{\bar{N}}\left(\sum_{i=1}^{k} f_{i}^{0} \otimes \varphi_{i}^{0}\right)-\epsilon .
$$

This completes the proof.

Lemma 3.3. If $N^{0}$ and $N^{00}$ denote two functions in $\mathfrak{A}\left(E_{1}, E_{2}\right)$ satisfying conditions I-IV and $N^{0} \leqq N^{00}$, then $\bar{N}^{0} \geqq \bar{N}^{00}$.

The proof is a simple consequence of Definition 3.2 and Lemma 3.1.

Consider the set $\mathfrak{A}^{*}\left(E_{1}, E_{2}\right)$ (Definition 1.3). Put $N(\bar{f})=N\left(\sum_{i=1}^{n} f_{i} \otimes \varphi_{i}\right)$ for $\sum_{i=1}^{n} f_{i} \otimes \varphi_{i}$ in $\bar{f} . N(\bar{f})$ is single-valued, as follows from IV for $N$. Conditions I-III tell us that $N(\bar{f})$ is a norm in $\mathfrak{A}^{*}\left(E_{1}, E_{2}\right)$.

Similarly, considering sets of equivalent expressions in $\mathfrak{A}\left(\bar{E}_{1}, \bar{E}_{2}\right)$ as new elements $\bar{F}$, we obtain a set $\mathfrak{A}^{*}\left(\bar{E}_{1}, \bar{E}_{2}\right)$ and a norm $\bar{N}(\bar{F})$ in $\mathfrak{A}^{*}\left(\bar{E}_{1}, \bar{E}_{2}\right) . \bar{N}(\bar{F})$ will be called the norm associate with $N$.

We complete $\mathfrak{A}^{*}\left(E_{1}, E_{2}\right)$ to $E_{1} \otimes E_{2}$ by adding new elements, namely all fundamental sequences (satisfying Cauchy's condition) of elemients in $\mathfrak{Y}^{*}\left(E_{1}, E_{2}\right)$ with the following conventions:

$(\alpha)$ An element $\bar{f}$ of $\mathfrak{A}^{*}\left(E_{1}, E_{2}\right)$ will be considered identical with the sequence $\bar{f}, \bar{f}, \bar{f}, \cdots$.

$(\beta)$ Two fundamental sequences

$$
\bar{f}_{1}^{(1)}, \bar{f}_{2}^{(1)}, \bar{f}_{3}^{(1)}, \ldots ; \quad \bar{f}_{1}^{(2)}, \bar{f}_{2}^{(2)}, \bar{f}_{3}^{(2)}, \ldots
$$


are considered identical if and only if

$$
\lim _{n \rightarrow \infty} N\left(\bar{f}_{n}^{(1)}-\bar{f}_{n}^{(2)}\right)=0 .
$$

$(\gamma)$ The norm of a fundamental sequence $\bar{f}_{1}, \bar{f}_{2}, \bar{f}_{3}, \ldots$ is by definition $\lim _{n \rightarrow \infty} N\left(\bar{f}_{n}\right)$.

In a similar way we complete $\mathfrak{A}^{*}\left(\bar{E}_{1}, \bar{E}_{2}\right)$ to $\bar{E}_{1} \otimes \bar{E}_{2}$.

From Lemma 2.1.c follows that $\left(\sum_{j=1}^{m} F_{j} \otimes \phi_{j}\right)\left(\sum_{i=1}^{m} f_{i} \otimes \varphi_{i}\right)$ depends only upon $\bar{F}$ resp. $\bar{f}$, for which $\sum_{j=1}^{m} F_{j} \otimes \phi_{j}$ resp. $\sum_{i=1}^{m} f_{i} \otimes \varphi_{i}$ are expressions $; \bar{F}(\bar{f})$ is therefore a uniquely defined number for $\bar{f}$ in $\mathfrak{A}^{*}\left(E_{1}, E_{2}\right), \bar{F}$ in $\mathfrak{A}^{*}\left(\bar{E}_{1}, \bar{E}_{2}\right)$.

LEMMA 3.4. If $\bar{F}_{1}, \bar{F}_{2}, \cdots$ resp. $\bar{f}_{1}, \bar{f}_{2}, \cdots$ denotes a fundamental sequence of elements in $\mathfrak{A}^{*}\left(\bar{E}_{1}, \bar{E}_{2}\right)$ resp. $\mathfrak{A}^{*}\left(E_{1}, E_{2}\right)$, then the sequence $\bar{F}_{1}\left(\bar{f}_{1}\right), \bar{F}_{2}\left(\bar{f}_{2}\right), \ldots$ is convergent.

The proof is immediate.

Without fear of misunderstanding, we shall also denote the elements of $E_{1} \otimes E_{2}$ resp. $\bar{E}_{1} \otimes \bar{E}_{2}$ by $\bar{f}$, resp. $\bar{F}$.

From Lemma 3.4 follows that $\bar{F}(\bar{f})$ is uniquely defined for $\bar{f}$ in $E_{1} \otimes E_{2}$, $\bar{F}$ in $\bar{E}_{1} \otimes \bar{E}_{2}$ and $|\bar{F}(\bar{f})| \leqq \bar{N}(\bar{F}) N(\bar{f})$.

Let $\bar{F}^{0}$ be an element of $\bar{E}_{1} \otimes \bar{E}_{2}$. It is a consequence of Lemma 3.4 that $\bar{F}^{0}(\bar{f})$ is a linear functional on $E_{1} \otimes E_{2}$. We shall write therefore

$$
\bar{E}_{1} \otimes \bar{E}_{2} \subset \overline{E_{1} \otimes E_{2}}\left({ }^{2}\right) .
$$

We shall assume further that $N$ defined on $\mathfrak{A}\left(E_{1}, E_{2}\right)$ in addition to I-IV satisfies also the following condition of continuity:

V. $N\left(\sum_{i=1}^{n} f_{i} \otimes \varphi_{i}\right)$ is a continuous function of the $f_{i}$ and $\varphi_{i}$, that is, if $\epsilon>0$ is given, we can find $a \delta=\delta\left(f_{1}, \cdots, f_{n} ; \varphi_{1}, \cdots, \varphi_{n}\right)>0$, such that for $\left\|f_{i}-f_{i}^{\prime}\right\|<\delta,\left\|\varphi_{i}-\varphi_{i}^{\prime}\right\|<\delta$, for $i=1, \cdots, n$, we have

$$
N\left(\sum_{i=1}^{n} f_{i} \otimes \varphi_{i} \cdot-\cdot \sum_{i=1}^{n} f_{i}^{\prime} \otimes \varphi_{i}^{\prime}\right)<\epsilon .
$$

Lemma 3.5. If $E_{1}, E_{2}$ are separable and $N$ satisfies conditions $\mathrm{I}-\mathrm{V}$, then $E_{1} \otimes E_{2}$ is separable.

Proof. Let $f_{1}^{0}, f_{2}^{0}, \cdots$ resp. $\varphi_{1}^{0}, \varphi_{2}^{0}, \cdots$ denote two sequences dense in $E_{1}$ resp. $E_{2}$. Then the set of expressions $\sum_{i=1}^{n} f_{t_{i}}^{0} \otimes \varphi_{i_{i}^{\prime}}^{0}, t_{i}, t_{i}^{\prime}=1,2,3, \cdots$; $n=1,2,3, \cdots$, is dense in $\mathfrak{A}\left(E_{1}, E_{2}\right)$. Hence the set of elements $\vec{f}$ for which these expressions stand is dense in $\mathfrak{A}^{*}\left(E_{1}, E_{2}\right)$ therefore also in $E_{1} \otimes E_{2}$.

Definition 3.3. A function $N$ of expressions in $\mathfrak{A}\left(E_{1}, E_{2}\right)$ is called a crossnorm if in addition to I-IV it satisfies the following "cross-property":

(2) A supplementary remark is made in Part A, $\$ 6$.

$$
N(f \otimes \varphi)=\|f\|\|\varphi\| \quad \text { for } f \in E_{1}, \varphi \in E_{2} .
$$


Lemma 3.6. A crossnorm $N$ satisfies condition V.

Proof. This is an immediate consequence of the following relation

$$
\begin{aligned}
N\left(\sum_{i=1}^{n} f_{i} \otimes \varphi_{i} \cdot-\cdot \sum_{i=1}^{n} f_{i}^{\prime} \otimes \varphi_{i}^{\prime}\right) \leqq N\left(\sum_{i=1}^{n}\left(f_{i}-f_{i}^{\prime}\right) \otimes \varphi_{i}\right) \\
\quad+N\left(\sum_{i=1}^{n} f_{i} \otimes\left(\varphi_{i}-\varphi_{i}^{\prime}\right)\right)+N\left(\sum_{i=1}^{n}\left(f_{i}-f_{i}^{\prime}\right) \otimes\left(\varphi_{i}-\varphi_{i}^{\prime}\right)\right) \\
\leqq \\
\sum_{i=1}^{n}\left\|f_{i}-f_{i}^{\prime}\right\|\left\|\varphi_{i}\right\|+\sum_{i=1}^{n}\left\|f_{i}\right\|\left\|\varphi_{i}-\varphi_{i}^{\prime}\right\|+\sum_{i=1}^{n}\left\|f_{i}-f_{i}^{\prime}\right\|\left\|\varphi_{i}-\varphi_{i}^{\prime}\right\| .
\end{aligned}
$$

Sometimes we shall assume that the norm $N$ satisfies the following condition :

VI. $\left\|\sum_{i=1}^{n} F\left(f_{i}\right) \varphi_{i}\right\| \leqq\|F\| N\left(\sum_{i=1}^{n} f_{i} \otimes \varphi_{i}\right)$ for $F$ in $\bar{E}_{1}$, and $\sum_{i=1}^{n} f_{i} \otimes \varphi_{i}$ in $\mathfrak{A}\left(E_{1}, E_{2}\right)$.

DEFINITION 3.4. From Lemma 2.1 follows that $\sum_{i=1}^{n} F\left(f_{i}\right) \varphi_{i}$ is invariant under equivalence. Let $\bar{f}$ be an element of $\mathfrak{A}^{*}\left(E_{1}, E_{2}\right)$ for which $\sum_{i=1}^{n} f_{i} \otimes \varphi_{i}$ is an expression. We define $T_{\bar{f}} F$ as the transformation from $\bar{E}_{1}$ to $E_{2}$ such that $T-F=\sum_{i=1}^{n} F\left(f_{i}\right) \varphi_{i}$.

LEMмa 3.7. If $T_{\bar{f}}=0$ then $\bar{f}=0$.

Proof. Let $\bar{f} \neq 0$ and $\sum_{1=1}^{n} f_{i} \otimes \varphi_{i}$ be an expression for $\bar{f}$ for which the $f_{i}$ 's and $\varphi_{i}$ 's are linearly independent. Thus $f_{1} \neq 0$. We can find an $F \subset \bar{E}_{1}$, such that $F\left(f_{1}\right) \neq 0$. This implies $\sum_{i=1}^{n} F\left(f_{i}\right) \varphi_{i} \neq 0$, since the $\varphi_{i}$ 's are linearly independent. Thus $T_{\bar{f}} F \neq 0$ and $T_{\bar{f}} \neq 0$. We have shown that $\bar{f} \neq 0$ implies $T_{\bar{f}} \neq 0$. This completes the proof.

Lemma 3.8. Condition II, IV, and VI for $N$ imply I.

Proof. From II and IV follows that $\sum_{i=1}^{n} f_{i} \otimes \varphi_{i} \simeq 0 \otimes 0$ implies $N\left(\sum_{i=1}^{n} f_{i} \otimes \varphi_{i}\right)=0$. Now let $N\left(\sum_{i=1}^{n} f_{i} \otimes \varphi_{i}\right)=0$. VI implies $\left\|\sum_{i=1}^{n} F\left(f_{i}\right) \varphi_{i}\right\|=0$ for $F$ in $\bar{E}_{1}$. Hence $\sum_{i=1}^{n} f_{i} \otimes \varphi_{i} \simeq 0 \otimes 0$ by Lemma 3.7.

4. Among the functions $N$, a particular one which we shall denote by $N_{1}$ will be of great interest to us.

Definition 4.1. Let $\sum_{i=1}^{m} f_{i}^{0} \otimes \varphi_{i}^{0}$ be a fixed expression. We define $N_{1}\left(\sum_{i=1}^{n} f_{i}^{0} \otimes \varphi_{i}^{0}\right)=\inf \sum_{i=1}^{n}\left\|f_{i}\right\|\left\|\varphi_{i}\right\|$ where inf (the greatest lower bound) is taken over the set of all expressions $\sum_{i=1}^{n} f_{i} \otimes \varphi_{i}$ equivalent to $\sum_{i=1}^{m} f_{i}^{0} \otimes \varphi_{i}^{0}$.

Lemma 4.1. $N_{1}$ satisfies conditions II, III, IV, and VI, therefore also I by Lemma 3.8; $N_{1}$ is a crossnorm, therefore it satisfies $\mathrm{V}$ as follows from Lemma 3.6.

Proof. That $N_{1}$ satisfies II and IV is obvious We proceed to prove III. Let $\sum_{i=1}^{m} f_{i}^{0} \otimes \varphi_{i}^{0}$ and $\sum_{i=1}^{n} g_{i}^{0} \otimes \psi_{i}^{0}$ be two given expressions, and let $\epsilon>0$ be given. 
Take an expression

such that

$$
\sum_{i=1}^{r} f_{i} \otimes \varphi_{i} \simeq \sum_{i=1}^{m} f_{i}^{0} \otimes \varphi_{i}^{0}
$$

$$
\sum_{i=1}^{r}\left\|f_{i}\right\|\left\|\varphi_{i}\right\| \leqq N_{1}\left(\sum_{i=1}^{m} f_{i}^{0} \otimes \varphi_{i}^{0}\right)+\epsilon / 2 .
$$

Similarly we find

such that

$$
\sum_{i=1}^{8} g_{i} \otimes \psi_{i} \simeq \sum_{i=1}^{n} g_{i}^{0} \otimes \psi_{i}^{0}
$$

We have

$$
\sum_{i=1}^{\dot{1}}\left\|g_{i}\right\|\left\|\psi_{i}\right\| \leqq N_{1}\left(\sum_{i=1}^{n} g_{i}^{0} \otimes \psi_{i}^{0}\right)+\epsilon / 2 \text {. }
$$

$$
\sum_{i=1}^{r} f_{i} \otimes \varphi_{i} \cdot+\cdot \sum_{i=1}^{8} g_{i} \otimes \psi_{i} \simeq \sum_{i=1}^{m} f_{i}^{0} \otimes \varphi_{i}^{0} \cdot+\cdot \sum_{i=1}^{n} g_{i}^{0} \otimes \mid \psi_{i}^{0}
$$

Condition IV and Definition 4.1 give

$$
\begin{aligned}
N_{1}\left(\sum_{i=1}^{m} f_{i}^{0} \otimes \varphi_{i}^{0} \cdot+\cdot \sum_{i=1}^{n} g_{i}^{0} \otimes \psi_{i}^{0}\right) & \leqq \sum_{i=1}^{r}\left\|f_{i}\right\|\left\|\varphi_{i}\right\|+\sum_{i=1}^{s}\left\|g_{i}\right\|\left\|\psi_{i}\right\| \\
& \leqq N_{1}\left(\sum_{i=1}^{m} f_{i}^{0} \otimes \varphi_{i}^{0}\right)+N_{1}\left(\sum_{i=1}^{n} g_{i}^{0} \otimes \psi_{i}^{0}\right)+\epsilon .
\end{aligned}
$$

This proves III.

We proceed to prove VI. Let $\sum_{i=1}^{n} f_{i} \otimes \varphi_{i}$ be an expression for $\bar{f}$. We have (Definition 3.4)

$$
\left\|T_{\jmath} F\right\|=\left\|\sum_{i=1}^{n} F\left(f_{i}\right) \varphi_{i}\right\| \leqq\|F\| \sum_{i=1}^{n}\left\|f_{i}\right\|\left\|\varphi_{i}\right\| .
$$

This holds for every expression in $\bar{f}$. Hence

$$
\left\|T_{j} F\right\| \leqq\|F\| \cdot \inf \sum_{i=1}^{n}\left\|f_{i}\right\|\left\|\varphi_{i}\right\|=\|F\| N_{1}(\bar{f}) .
$$

To prove the cross-property, we assume $f \neq 0$ and $\varphi \neq 0$ (for $f=0$ or $\varphi \doteq 0$, the proof is obvious). Let $F \in \bar{E}_{1}$ be such that $F(f)=\|f\|,\|F\|=1$. If $\sum_{i=1}^{n} f_{i} \otimes \varphi_{i} \simeq f \otimes \varphi$, we have (Lemma 2.1)

$$
\|F(f) \varphi=\| \sum_{i=1}^{n} F\left(f_{i}\right) \varphi_{i} \| \text { or }\|f\|\|\varphi\| \leqq \sum_{i=1}^{n}\left\|f_{i}\right\|\left\|\varphi_{i}\right\| .
$$

Therefore $N_{1}(f \otimes \varphi)=\|f\|\|\varphi\|$. This completes the proof.

LEMMA 4.2. $N_{1}$ is the greatest crossnorm. 
Proof. For any crossnorm $N$, we have

$$
N\left(\sum_{i=1}^{k} f_{i}^{0} \otimes{\stackrel{\varphi}{\varphi_{i}}}^{0}\right) \leqq \sum_{i=1}^{k} N\left(f_{i}^{0} \otimes{\stackrel{\varphi}{\varphi_{i}}}^{0}\right)=\sum_{i=1}^{k}\left\|f_{i}^{0}\right\|\left\|\varphi_{i}^{0}\right\| .
$$

Condition IV for $N$ implies

$$
N\left(\sum_{i=1}^{k} f_{i}^{0} \otimes \varphi_{i}^{0}\right) \leqq \inf \sum_{i=1}^{n}\left\|f_{i}\right\|\left\|\varphi_{i}\right\|=N_{1}\left(\sum_{i=1}^{k} f_{i}^{0} \otimes \varphi_{i}^{0}\right)
$$

where inf is taken over the set of all expressions $\sum_{i=1}^{n} f_{i} \otimes \varphi_{i}$ equivalent to $\sum_{i=1}^{k} f_{i}^{0} \otimes \varphi_{i}^{0}$.

LEMMA 4.3. The norm $\bar{N}$ associated with a crossnorm $N$, satisfies the condition $\bar{N}(F \otimes \phi) \geqq\|F\|\|\phi\|$ for $F \subset \bar{E}_{1}, \phi \subset \bar{E}_{2}$.

The proof is immediate.

Definition 4.2. We define $N_{0}$ :

$$
N_{0}\left(\sum_{i=1}^{n} f_{i} \otimes \varphi_{i}\right)=\sup \left(\left|\sum_{i=1}^{n} F\left(f_{i}\right) \phi\left(\varphi_{i}\right)\right| /\|F\|\|\phi\|\right)
$$

where sup (the least upper bound) is taken over the set of numbers obtained when $F$ resp. $\phi$ varies in $\bar{E}_{1}$ resp. $\bar{E}_{2}$.

LEMMA 4.4. $N_{0}$ and $\bar{N}_{0}$ are crossnorms.

Proof. It is not difficult to verify that $N_{0}$ is a crossnorm. We shall prove that $\bar{N}_{0}$ is a crossnorm. We have

$$
\begin{aligned}
\left(\mid\left(F^{0}\right.\right. & \left.\left.\otimes \phi^{0}\right)\left(\sum_{i=1}^{n} f_{i} \otimes \varphi_{i}\right) \mid / N_{0}\left(\sum_{i=1}^{n} f_{i} \otimes \varphi_{i}\right)\right) \\
& \leqq\left|\left(F^{0} \otimes \phi^{0}\right)\left(\sum_{i=1}^{n} f_{i} \otimes \varphi_{i}\right)\right|\left(\left\|F^{0}\right\|\left\|\phi^{0}\right\| /\left|\left(F^{0} \otimes \phi^{0}\right)\left(\sum_{i=1}^{n} f_{i} \otimes \varphi_{i}\right)\right|\right) \\
& =\left\|F^{0}\right\|\left\|\phi^{0}\right\| .
\end{aligned}
$$

Hence (Definition 3.2) $\bar{N}_{0}\left(F^{0} \otimes \phi^{0}\right) \leqq\left\|F^{0}\right\|\left\|\phi^{0}\right\|$. This together with Lemma 4.3 concludes the proof.

LEMMA 4.5. The associate $\bar{N}$ with a crossnorm $N \geqq N_{0}$ is also a crossnorm.

Proof. Lemma 3.3 gives $\bar{N} \leqq \bar{N}_{0}$. In particular $\bar{N}(F \otimes \phi) \leqq \bar{N}_{0}(F \otimes \phi)$ $=\|F\|\|\phi\|$ by Lemma 4.4. An application of Lemma 4.3 concludes the proof.

THEOREM 4.1. The associate $\bar{N}$ with a crossnorm $N$ is also a crossnorm if and only if $N \geqq N_{0} ; N_{0}$ is therefore the least crossnorm whose associate is also a crossnorm. 
Proof. The sufficiency is proved in Lemma 4.5. We shall prove the necessity. Suppose that for a crossnorm $N$ and a certain expression $\sum_{i=1}^{n} f_{i}^{0} \otimes \varphi_{i}^{0}$ we have

$$
N\left(\sum_{i=1}^{n} f_{i}^{0} \otimes \varphi_{i}^{0}\right)<N_{0}\left(\sum_{i=1}^{n} f_{i}^{0} \otimes \varphi_{i}^{0}\right)
$$

Then, there exists an $F^{0} \in \bar{E}_{1}$ and $\phi^{\circ} \in \bar{E}_{2}$ such that

$$
N\left(\sum_{i=1}^{n} f_{i}^{0} \otimes \varphi_{i}^{0}\right)<\left|\sum_{i=1}^{n} F^{0}\left(f_{i}^{0}\right) \phi^{0}\left(\varphi_{i}^{0}\right)\right| /\left\|F^{0}\right\|\left\|\phi^{0}\right\|
$$

and consequently

$$
\bar{N}\left(F^{0} \otimes \phi^{0}\right) \geqq\left|\left(F^{0} \otimes \phi^{0}\right)\left(\sum_{i=1}^{n} f_{i}^{0} \otimes \varphi_{i}^{0}\right)\right| / N\left(\sum_{i=1}^{n} f_{i}^{0} \otimes \phi_{i}^{0}\right)>\left\|F^{0}\right\|\left\|\phi^{0}\right\|
$$

or $\bar{N}$ is not a crossnorm. This completes the proof $\left({ }^{3}\right)$.

THEOREM 4.1.1. A crossnorm $N$ satisfies condition VI if and only if its associate $\bar{N}$ is also a crossnorm.

The proof is similar to that in Theorem 4.1.

In our future work, speaking about "the least crossnorm," we shall have in mind "the least crossnorm whose associate is also a crossnorm," namely $N_{0}$.

5. The least crossnorm $N_{0}$, as well as the greatest crossnorm $N_{1}$, are defined for any two Banach spaces $E_{1}, E_{2}$. For this reason we shall call them general crossnorms. Similarly $(1 / 2)\left(N_{0}+N_{1}\right), \overline{(1 / 2)\left(N_{0}+N_{1}\right)}, \cdots$ are general crossnorms $\left({ }^{4}\right)$.

Let $K$ denote the smallest class of crossnorms satisfying the following conditions:

1. $N_{1} \in K$.

2. If $N \in K$, then $\bar{N} \in K$.

3. If $N^{0}$ and $N^{00}$ belong to $K$, then $a N^{0}+(1-a) N^{00}$ belongs to $K$ for $0<a<1$.

4. If $N^{0}, N^{00}, N^{000}, \cdots$ denotes a monotonic sequence of crossnorms belonging to $K$, then its limit (which exists because the sequence is bounded by the least and greatest crossnorms) also is in $K$.

Definition 5.1. Crossnorms in $K$ are defined for any two Banach spaces $E_{1}, E_{2}$. For this reason we shall call them general crossnorms.

Lemma 5.1. If $N^{(1)}$ and $N^{(2)}$ denote two crossnorms (not necessarily general) and $a, b$ real numbers, such that $a+b=1,0<a<1$, then

$\left({ }^{3}\right)$ An immediate problem is mentioned in Part B, $\$ 6$.

(4) For a general crossnorm $N, \bar{N}$ is to have the following significance in $\mathfrak{A}^{*}\left(E_{1}, E_{2}\right)$. We consider $N$ on $\mathscr{U}^{*}\left(\bar{E}_{1}, \bar{E}_{2}\right)$. For this there is an $\bar{N}$ defined on $\mathfrak{Q}^{*}\left(\overline{\bar{E}}_{1}, \overline{\bar{E}}_{2}\right)$. We consider the latter confined to $\mathfrak{A}^{*}\left(E_{1}, E_{2}\right) \subseteq \mathfrak{Y}^{*}\left(\overline{\bar{E}}_{1}, \overline{\bar{E}}_{2}\right)$. 


$$
\overline{a N^{(1)}+b N^{(2)}} \leqq a \bar{N}^{(1)}+b \bar{N}^{(2)} \text {. }
$$

Proof. We shall prove that for any $\bar{F}_{0} \in \mathfrak{A} *\left(\bar{E}_{1}, \bar{E}_{2}\right)$ we have

$$
\sup \frac{\left|\bar{F}_{0}(\bar{f})\right|}{a N^{(1)}(\bar{f})+b N^{(2)}(\bar{f})} \leqq a \sup \frac{\left|\bar{F}_{0}(\bar{f})\right|}{N^{(1)}(\bar{f})}+b \sup \frac{\left|\bar{F}_{0}(\bar{f})\right|}{N^{(2)}(\bar{f})}
$$

where sup is taken over all $\bar{f}^{\prime} \mathrm{s}(\neq 0)$ in $\mathfrak{Q}^{*}\left(E_{1}, E_{2}\right)$. Suppose that the contrary holds, that is, for a certain $\bar{F}_{0}$ the last inequality does not hold. Then for a certain $\bar{f}_{0}$ in $\mathfrak{A}^{*}\left(E_{1}, E_{2}\right)$ we have

$$
\frac{\left|\bar{F}_{0}\left(\bar{f}_{0}\right)\right|}{a N^{(1)}\left(\bar{f}_{0}\right)+b N^{(2)}\left(\bar{f}_{0}\right)}>a \frac{\left|\bar{F}_{0}\left(\bar{f}_{0}\right)\right|}{N^{(1)}\left(\bar{f}_{0}\right)}+b \frac{\left|\bar{F}_{0}\left(\bar{f}_{0}\right)\right|}{N^{(2)}\left(\bar{f}_{0}\right)} .
$$

This gives: $\left\{N^{(1)}\left(\bar{f}_{0}\right)-N^{(2)}\left(\bar{f}_{0}\right)\right\}^{2}<0$. This cannot happen, and therefore the proof is completed.

Corollary. Let $N^{0}$ and $N^{00}$ denote two general crossnorms, and $a, b$ real numbers, such that $a+b=1,0<a<1$, then

$$
\overline{a N^{0}+b N^{00}} \leqq a \bar{N}^{0}+b \bar{N}^{00} \text {. }
$$

LEMma 5.2. If $N$ denotes a general crossnorm and $\bar{N}$ its associate, then putting $N^{(a)}=a N+(1-a) \bar{N}, 0 \leqq a \leqq 1$, we have $N^{(a)}+\bar{N}^{(a)} \leqq N+\bar{N}\left({ }^{(5)}\right.$.

Proof. For $a=1$ the statement is trivial, For $a=0, \bar{N}+\overline{\bar{N}} \leqq \bar{N}+N$ as follows from Lemma 3.2. For $0<a<1$, Lemma 5.1 gives $N^{(a)}+\bar{N}^{(a)}$ $\leqq\{a N+(1-a) \bar{N}\}+\{a \bar{N}+(1-a) \overline{\bar{N}}\} \leqq N+\bar{N}$ as follows from Lemma 3.2.

CoRollary. For a general crossnorm $N$, we have $\overline{(1 / 2)(N+\bar{N})}$ $\leqq(1 / 2)(N+\bar{N})$.

Proof. $N^{(1 / 2)}+\bar{N}^{(1 / 2)} \leqq N+\bar{N}=2 N^{(1 / 2)}($ Lemma 5.2$)$. Hence $\bar{N}^{(1 / 2)} \leqq N^{(1 / 2)}$. This completes the proof.

We shall show an immediate application of the last result.

THEOREM 5.1. There exists a general crossnorm $N$, with the following properties:

$\alpha . N$ is identical with its associate $\bar{N}$.

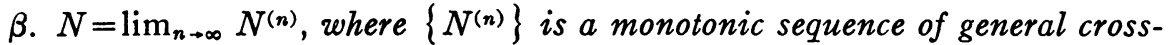
norms defined in the following way: Let $N_{1}$ denote the greatest crossnorm. Put $N^{(1)}=(1 / 2)\left(N_{1}+\bar{N}_{1}\right)$. Let $n>1$, and suppose we have defined $N^{(k)}$ for $k<n$, then put $N^{(n)}=(1 / 2)\left(N^{(n-1)}+\bar{N}^{(n-1)}\right)$.

Proof. Since $N_{1}$ is the greatest crossnorm, its associate $\bar{N}_{1}$ is also a crossnorm (Lemma 4.5), and therefore $\bar{N}_{1} \leqq N_{1}$. Lemma 3.3 and the corollary to Lemma 5.2 give

(5) For simplicity of notation $\bar{N}^{(a)}$ shall denote the associate with $N^{(a)}$. 


$$
\bar{N}_{1} \leqq \bar{N}^{(1)} \leqq N^{(1)} \leqq N_{1} .
$$

The last inequality proves that $\bar{N}^{(1)}$ is a crossnorm, therefore $N^{(2)}$ is also a crossnorm. Similarly we obtain

$$
\bar{N}_{1} \leqq \bar{N}^{(1)} \leqq \bar{N}^{(2)} \leqq N^{(2)} \leqq N^{(1)} \leqq N_{1} .
$$

This proves that $\bar{N}^{(2)}$ is also a crossnorm, therefore $N^{(3)}$ is a crossnorm. Repeating the same process, we obtain a decreasing sequence of crossnorms

$$
N_{1} \geqq N^{(1)} \geqq N^{(2)} \geqq \cdots
$$

and an increasing sequence of its associate crossnorms

$$
\bar{N}_{1} \leqq \bar{N}^{(1)} \leqq \bar{N}^{(2)} \leqq \cdots .
$$

The first sequence is bounded from below by $\bar{N}_{1}$, hence is convergent; let $N$ denote its limit. The second sequence is bounded from above by $N_{1}$, hence convergent; let $\mathfrak{N}$ denote its limit. Therefore:

(1) $\lim _{n \rightarrow \infty} N^{(n)}=N$; $\lim _{n \rightarrow \infty} \bar{N}^{(n)}=\mathfrak{N}$.

It is easy to verify that

$$
N^{(n)}-\bar{N}^{(n)} \leqq\left(1 / 2^{n}\right)\left(N_{1}-\bar{N}_{1}\right) \quad \text { for } n=1,2, \cdots
$$

bécause

$$
\begin{aligned}
N^{(1)}-\bar{N}^{(1)} & =(1 / 2)\left(N_{1}+\bar{N}_{1}\right)-\bar{N}^{(1)} \leqq(1 / 2)\left(N_{1}+\bar{N}_{1}\right)-\bar{N}_{1} \\
& =(1 / 2)\left(N_{1}-\bar{N}_{1}\right), \\
N^{(2)}-\bar{N}^{(2)} & =(1 / 2)\left(N^{(1)}+\bar{N}^{(1)}\right)-\bar{N}^{(2)} \leqq(1 / 2)\left(N^{(1)}+\bar{N}^{(1)}\right)-\bar{N}^{(1)} \\
& =(1 / 2)\left(N^{(1)}-\bar{N}^{(1)}\right) \leqq\left(1 / 2^{2}\right)\left(N_{1}-\bar{N}_{1}\right),
\end{aligned}
$$

Therefore: $N=\mathfrak{R}$. We shall prove $N=\bar{N}$. Since $N \leqq N^{(n)}$ for $n=1,2, \cdots$ $\bar{N} \geqq \bar{N}^{(n)}$ hence (1) gives $\bar{N} \geqq \mathfrak{N}$ or

(2) $\bar{N} \geqq N$.

On the other hand,

$$
\bar{N}^{(n)} \leqq \mathfrak{R}=N \quad \text { for } n=1,2, \cdots ;
$$

hence $\overline{\bar{N}}^{(n)} \geqq \bar{N}$ and therefore (Lemma 3.2) $N^{(n)} \geqq \bar{N}$. (1) gives

(3) $N \geqq \bar{N}$.

(2) and (3) give the required result $\left({ }^{6}\right)$.

TheOREM 5.1.1. Let $N^{0}$ denote a general crossnorm (Definition 5.1). The same construction (which we have applied to $N_{1}$ in Theorem 5.1) applied to $N^{0}$, will always lead to a crossnorm $\dot{N}$ satisfying conditions $\alpha, \beta$ mentioned in Theorem 5.1.

(6) The immediate problem is mentioned in Part C, $\$ 6$. 
Proof. Let us notice that for a general crossnorm $N^{0}$, we have always $\bar{N}_{1} \leqq N^{0} \leqq N_{1}$, hence also $\bar{N}_{1} \leqq \bar{N}^{0} \leqq N_{1}$ by virtue of Lemmas 3.2 and 3.3. This assures that the associate with a general crossnorm is also a crossnorm. We put $N^{(1)}=(1 / 2)\left(N^{0}+\bar{N}^{0}\right), N^{(1)}$ is a crossnorm and $\bar{N}^{(1)} \leqq N^{(1)}($ Lemma 5.2) is also a crossnorm. We have therefore the situation mentioned in Theorem 5.1. Let $n>1$, and suppose we have defined $N^{(k)}$ for $k<n$, then put $N^{(n)}=(1 / 2)\left(N^{(n-1)}+\bar{N}^{(n-1)}\right)$. As in Theorem 5.1 we prove that $\lim _{n \rightarrow \infty} N^{(n)}$ exists; call it $N$, and $\lim _{n \rightarrow \infty} \bar{N}^{(n)}$ exists; call it $\mathfrak{N}$. Further,

$$
N^{(n)}-\bar{N}^{(n)} \leqq\left(1 / 2^{n-1}\right)\left(N^{(1)}-\bar{N}^{(1)}\right) \quad \text { for } n=1,2, \cdots \text {; }
$$

hence $N=\mathfrak{R}$. Finally we prove (in exactly the same way as in Theorem 5.1 ) that $\bar{N}=N$.

We shall apply our results to Hilbert spaces. Let $E_{1}, E_{2}$, denote two Hilbert spaces, and $N$ a crossnorm in $\mathfrak{A}\left(E_{1}, E_{2}\right)$ (Definition 1.3).

DEFINITION 5.2. $N$ will be termed self-associate if for every expression $\sum_{j=1}^{m} F_{j} \otimes \phi_{j}$ in $\mathfrak{A}\left(\bar{E}_{1}, \bar{E}_{2}\right)$, we have

$$
\bar{N}\left(\sum_{j=1}^{m} F_{j} \otimes \phi_{j}\right)=N\left(\sum_{j=1}^{m} f_{j} \otimes \varphi_{j}\right),
$$

where $f_{j}$ resp. $\varphi_{j}$ is the element in $E_{1}$ resp. $E_{2}$ for which $F_{j}(f) \equiv\left(f, f_{j}\right)$ in $E_{1}$ resp. $\phi_{j}(\varphi) \equiv\left(\varphi, \varphi_{j}\right)$ in $E_{2}$.

For two Hilbert spaces $E_{1}, E_{2}$, F. J. Murray and J. von Neumann introduce the following crossnorm in $\mathfrak{A}\left(E_{1}, E_{2}\right)$ [10]:

$$
N\left(\sum_{j=1}^{n} f_{j} \otimes \varphi_{j}\right)=\left\{\sum_{i=1}^{n} \sum_{k=1}^{n}\left(f_{i}, f_{k}\right)\left(\varphi_{i}, \varphi_{k}\right)\right\}^{1 / 2}
$$

where the symbol $\left(f_{i}, f_{k}\right)$ denotes the inner product. Let $\bar{N}$ denote the associate with $N$, and let

$$
\sum_{j=1}^{m} F_{j}^{0} \otimes \phi_{j}^{0}
$$

be a fixed expression in $\mathfrak{A}\left(\bar{E}_{1}, \bar{E}_{2}\right)$. We have

$$
\begin{aligned}
& \bar{N}\left(\sum_{j=1}^{m} F_{j}^{0} \otimes \phi_{j}^{0}\right) \\
& =\sup \left|\left(\sum_{j=1}^{m} F_{j}^{0} \otimes \phi_{j}^{0}\right)\left(\sum_{i=1}^{n} f_{i} \otimes \varphi_{i}\right)\right| / N\left(\sum_{i=1}^{n} f_{i} \otimes \varphi_{i}\right) \\
& =\sup \left|\left(\sum_{j=1}^{m} \sum_{i=1}^{n}\left(f_{i}, f_{j}^{0}\right)\left(\varphi_{i}, \varphi_{j}^{0}\right)\right)\right| /\left\{\sum_{i=1}^{n} \sum_{k=1}^{n}\left(f_{i}, f_{k}\right)\left(\varphi_{i}, \varphi_{k}\right)\right\}^{1 / 2}
\end{aligned}
$$

where $F_{j}^{0}(f) \equiv\left(f, f_{j}^{0}\right), \phi_{j}^{0}(\varphi) \equiv\left(\varphi, \varphi_{j}^{0}\right)$. Applying Schwarz's inequality to the numerator, we get 


$$
\begin{gathered}
\bar{N}\left(\sum_{j=1}^{m} F_{j}^{0} \otimes \phi_{j}^{0}\right) \\
\leqq \sup \left(\left\{\sum_{j=1}^{m} \sum_{l=1}^{m}\left(f_{j}^{0}, f_{l}^{0}\right)\left(\varphi_{i}^{0}, \varphi_{l}^{0}\right)\right\}^{1 / 2}\left\{\sum_{i=1}^{n} \sum_{k=1}^{n}\left(f_{i}, f_{k}\right)\left(\varphi_{i}, \varphi_{k}\right)\right\}^{1 / 2}\right. \\
\\
\left./\left\{\sum_{i=1}^{n} \sum_{k=1}^{n}\left(f_{i}, f_{k}\right)\left(\varphi_{i}, \varphi_{k}\right)\right\}^{1 / 2}\right)
\end{gathered}
$$

or

$$
\bar{N}\left(\sum_{j=1}^{m} F_{j}^{0} \otimes \phi_{j}^{0}\right) \leqq N\left(\sum_{j=1}^{m} f_{j}^{0} \otimes \varphi_{j}^{0}\right) .
$$

On the other hand, taking in particular $\sum_{j=1}^{m} f_{j}^{0} \otimes \varphi_{j}^{0}$ for the variable expression $\sum_{i=1}^{n} f_{i} \otimes \varphi_{i}$, we get from (1)

$$
\begin{aligned}
& \bar{N}\left(\sum_{j=1}^{m} F_{j}^{0} \otimes \phi_{l}^{0}\right) \geqq\left|\sum_{j=1}^{m} \sum_{l=1}^{m}\left(f_{j}^{0}, f_{l}^{0}\right)\left(\varphi_{j}^{0}, \varphi_{l}^{0}\right)\right| /\left\{\sum_{j=1}^{m} \sum_{l=1}^{m}\left(f_{j}^{0}, f_{l}^{0}\right)\left(\varphi_{j}^{0}, \varphi_{l}^{0}\right)\right\}^{1 / 2} \\
& =\left\{\sum_{j=1}^{m} \sum_{l=1}^{m}\left(f_{j}^{0}, f_{l}^{0}\right)\left(\dot{\varphi}_{j}^{0}, \varphi_{l}^{0}\right)\right\}^{1 / 2} \text {. }
\end{aligned}
$$

This means $\bar{N}\left(\sum_{j=1}^{m} F_{j}^{0} \otimes \phi_{j}^{0}\right) \geqq N\left(\sum_{j-1}^{m} f_{j}^{0} \otimes \varphi_{j}^{0}\right)$. The last inequality together with (2) proves that

$$
\bar{N}\left(\sum_{j=1}^{m} F_{j}^{0} \otimes \phi_{j}^{0}\right)=N\left(\sum_{j=1}^{m} f_{j}^{0} \otimes \varphi_{j}^{0}\right)
$$

or the crossnorm introduced by F. J. Murray and J. von Neumann is selfassociate in the sense of Definition 5.2.

We shall denote this crossnorm by $S_{M, N}$.

THEOREM 5.2. If $E_{1}$ and $E_{2}$ denote two Hilbert spaces, then every self-associate crossnorm in $\mathfrak{A}\left(E_{1}, E_{2}\right)$ is identical with $S_{M, N}$.

Proof. Let $N$ be a crossnorm in $\mathfrak{A}\left(E_{1}, E_{2}\right)$. Since $\bar{E}_{1}=E_{1}, \bar{E}_{2}=E_{2}$, for an expression $\sum_{j=1}^{m} F_{j}^{0} \otimes \phi_{j}^{0}$ in $\mathfrak{A}\left(\bar{E}_{1}, \bar{E}_{2}\right)$ we have

$$
\begin{aligned}
\bar{N}\left(\sum_{j=1}^{m} F_{i}^{0} \otimes \phi_{j}^{0}\right) & =\bar{N}\left(\sum_{j=1}^{m} f_{j}^{0} \otimes{\stackrel{0}{\varphi_{j}}}^{0}\right) \\
& =\sup \left(\left|\sum_{j=1}^{m} \sum_{i=1}^{n}\left(f_{i}, f_{j}^{0}\right)\left(\varphi_{i}, \varphi_{j}^{0}\right)\right| / N\left(\sum_{i=1}^{n} f_{i} \otimes \varphi_{i}\right)\right)
\end{aligned}
$$

where $F_{j}^{0}(f) \equiv\left(f, f_{j}^{0}\right)$ in $E_{1}, \phi_{j}^{0}(\varphi) \equiv\left(\varphi, \varphi_{j}^{0}\right)$ in $E_{2}$. Taking in particular $\sum_{j=1}^{m} f_{j}^{0} \otimes \varphi_{j}^{0}$ for the variable expression $\sum_{j=1}^{n} f_{i} \otimes \varphi_{i}$ we get

$$
\bar{N}\left(\sum_{j=1}^{m} f_{j}^{0} \otimes \varphi_{j}^{0} N\left(\sum_{j=1}^{m} f_{j}^{0} \otimes \varphi_{j}^{0}\right) \geqq\left\{S_{M, N}\left(\sum_{j=1}^{m} f_{j}^{0} \otimes \varphi_{j}^{0}\right)\right\}^{2}\right.
$$


In particular if $N$ is self-associate in the sense of Definition 5.2, that is, $\bar{N}\left(\sum_{j=1}^{m} f_{j}^{0} \otimes \varphi_{j}^{0}\right)=N\left(\sum_{j=1}^{m} f_{j}^{0} \otimes \varphi_{j}^{0}\right)$ we get

$$
N^{2} \geqq S_{M, N}^{2} \quad \text { or } \quad N \geqq S_{M, N} \text {. }
$$

Taking the associate crossnorms for both sides of the last inequality, we get $\bar{N} \leqq \bar{S}_{M, N}$, or since $N$ and $S_{M, N}$ are self-associate $N \leqq S_{M, N}$. This gives $N=S_{M, N}$, and the proof is completed.

Theorem 5.3. If $E_{1}$ and $E_{2}$ denote two Hilbert spaces, then the general crossnorm $N$ constructed in Theorem 5.1 by means of the greatest crossnorm $N_{1}$ coincides with the usual self-associate crossnorm $S_{M, N}$.

Proof. From the construction of $N$, and $\bar{E}_{1}=E_{1}, \bar{E}_{2}=E_{2}$ obviously follows that $N$ is self-associate in the sense of Definition 5.2. Thus, $N$ is identical with $S_{M, N}$ by Theorem 5.2.

6 . In this section we present some remarks about the work of the preceding sections.

A. As a consequence of Lemma 3.4, $\bar{E}_{1} \otimes \bar{E}_{2} \subset \overline{E_{1} \otimes E_{2}}$ has been proved. It does not appear to be a simple matter to describe the exact conditions imposed upon a crossnorm, under which the relation $\bar{E}_{1} \otimes \bar{E}_{2}=\overline{E_{1} \otimes E_{2}}$ holds.

B. We have proved the existence of the least crossnorm, whose associate is also a crossnorm. We did not settle, however, whether the associate with every crossnorm is also a crossnorm, or there exist crossnorms whose associates are not crossnorms $\left({ }^{7}\right)$.

C. The general crossnorm $N$, constructed in Theorem 5.1 by means of the greatest crossnorm $N_{1}$, we are justified to term self-associate (extending hereby Definition 5.2). Theorem 5.1.1 states that the same construction applied to any general crossnorm will always lead to a self-associate crossnorm. We did not settle, however, the problem of "uniqueness," that is, whether the same construction applied to two different general crossnorms will always lead to the same self-associate crossnorm.

In connection with the question of uniqueness let us mention the following problem.

Let $N^{0}$ and $N^{00}$ denote two general crossnorms, and $N^{0} \leqq N^{00}$. Under what conditions is $N^{0}+\bar{N}^{0} \geqq N^{00}+\bar{N}^{00}$ ?

7. The following crossnorms are worth mentioning.

Definition 7.1. Let $\sum_{i=1}^{m} f_{i}^{0} \otimes \varphi_{i}^{0}$ be in $\mathfrak{A}\left(E_{1}, E_{2}\right)$. We put $N\left(\sum_{i=1}^{m} f_{i}^{0} \otimes \varphi_{i}^{0}\right)$ $=\inf \left(\max _{e_{i}= \pm 1}\left\|\sum_{i=1}^{n} \epsilon_{i}\right\| f_{i}\left\|\varphi_{i}\right\|\right)$ where inf (that is, the greatest lower bound) is taken over the set of all expressions $\sum_{i=1}^{n} f_{i} \otimes \varphi_{i}$ equivalent to $\sum_{i=1}^{m} f_{i}^{0} \otimes \varphi_{i}^{0}$.

THEOREM 7.1. $N$ in Definition 7.1 is a crossnorm.

(7) Added in proof: The author has since shown that if $E_{1}$ and $E_{2}$ are reflexive, that is, $\bar{E}_{i}=E_{i}$, then the associate of every crossnorm is also a crossnorm. 
Proof. It is obvious that $N$ satisfies II and IV of Definition 3.1. We shall prove III. For two given expressions $\sum_{i=1}^{m} f_{i} \otimes \varphi_{i}, \sum_{i=1}^{n} g_{i} \otimes \psi_{i}$ and a given $\epsilon>0$, we can find two expressions $\sum_{i=1}^{k} f_{i}^{\prime} \otimes \varphi_{i}^{\prime}, \sum_{i=1}^{r} g_{i}^{\prime} \otimes \psi_{i}^{\prime}$ such that

$\sum_{i=1}^{k} f_{i}^{\prime} \otimes \varphi_{i}^{\prime} \simeq \sum_{i=1}^{m} f_{i} \otimes \varphi_{i} ; \quad \max _{\epsilon_{i}= \pm 1}\left\|\sum_{i=1}^{k} \epsilon_{i}\right\| f_{i}^{\prime}\left\|\varphi_{i}^{\prime}\right\| \leqq N\left(\sum_{i=1}^{m} f_{i} \otimes \varphi_{i}\right)+\epsilon / 2$,

$\sum_{i=1}^{r} g_{i}^{\prime} \otimes \psi_{i}^{\prime} \simeq \sum_{i=1}^{n} g_{i} \otimes \psi_{i} ; \quad \max _{\epsilon_{i}= \pm 1}\left\|\sum_{i=1}^{r} \epsilon_{i}\right\| g_{i}^{\prime}\left\|\psi_{i}^{\prime}\right\| \leqq N\left(\sum_{i=1}^{n} g_{i} \otimes \psi_{i}\right)+\epsilon / 2$.

Let $\epsilon_{i}^{\prime}= \pm 1, i=1, \cdots, k ; \eta_{j}^{\prime}= \pm 1, j=1, \cdots, r$, be chosen so that

$\left\|\sum_{i=1}^{k} \epsilon_{i}^{\prime}\right\| f_{i}^{\prime}\left\|\varphi_{i}^{\prime}+\sum_{j=1}^{r} \eta_{i}^{\prime}\right\| g_{j}^{\prime}\left\|\psi_{j}^{\prime}\right\|=\max _{\epsilon_{i}= \pm 1 ; \eta_{j= \pm}}\left\|\sum_{i=1}^{k} \epsilon_{i}\right\| f_{i}^{\prime}\left\|\varphi_{i}^{\prime}+\sum_{i=1}^{r} \eta_{j}\right\| g_{j}^{\prime}\left\|\psi_{j}^{\prime}\right\|$

then IV and Definition 7.1 give

$$
\begin{aligned}
N\left(\sum_{i=1}^{m} f_{i} \otimes \varphi_{i} \cdot+\cdot \sum_{i=1}^{n} g_{i} \otimes \psi_{i}\right) \leqq\left\|\sum_{i=1}^{k} \epsilon_{i}^{\prime}\right\| f_{i}^{\prime}\left\|\varphi_{i}^{\prime}+\sum_{j=1}^{r} \eta_{i}^{\prime}\right\| g_{j}^{\prime}\left\|\psi_{j}^{\prime}\right\| \\
\leqq \max _{\epsilon_{i}= \pm 1}\left\|\sum_{i=1}^{k} \epsilon_{i}\right\| f_{i}^{\prime}\left\|\varphi_{i}^{\prime}\right\|+\max _{\epsilon_{i}= \pm 1}\left\|\sum_{i=1}^{r} \epsilon_{i}\right\| g_{i}^{\prime}\left\|\psi_{i}^{\prime}\right\| \\
\leqq N\left(\sum_{i=1}^{m} f_{i} \otimes \varphi_{i}\right)+N\left(\sum_{i=1}^{n} g_{i} \otimes \psi_{i}\right)+\epsilon .
\end{aligned}
$$

This proves III. We shall prove VI

$$
\left\|\sum_{i=1}^{n} F\left(f_{i}\right) \varphi_{i}\right\| \leqq \max _{\eta_{i}= \pm 1}\left\|\sum_{i=1}^{n} \eta_{i}\left|F\left(f_{i}\right)\right| \varphi_{i}\right\| \text {. }
$$

Let $\eta_{1}^{\prime}, \cdots, \eta_{n}^{\prime}$ denote that system of numbers $1,-1$, for which the right side of the last inequality is a maximum. Then,

$$
\left\|\sum_{i=1}^{n} F\left(f_{i}\right) \varphi_{i}\right\| \leqq\left\|\sum_{i=1}^{n} \eta_{i}^{\prime}\right\| F\|\| f_{i}\left\|\varphi_{i}\right\| \leqq\|F\| \cdot \max _{\epsilon_{i}= \pm 1}\left\|\sum_{i=1}^{n} \epsilon_{i}\right\| f_{i}\left\|\varphi_{i}\right\|
$$

and the proof of VI is a simple consequence of Lemma 2.1. Lemma $3.8 \mathrm{im}$ plies, therefore, I for $N$.

We complete the proof of the theorem by showing that $N$ has the crossproperty. Let $f \neq 0, \varphi \neq 0$ and $\sum_{i=1}^{n} f_{i} \otimes \varphi_{i} \simeq f \otimes \varphi$. Choose an $F \subset \bar{E}_{1}$ such that $F(f)=\|f\|,\|F\|=1$. Lemma 2.1 gives

or

$$
\|f\|\|\varphi\|=\|F(f) \varphi\|=\left\|\sum_{i=1}^{n} F\left(f_{i}\right) \varphi_{i}\right\| \leqq\|F\| \max _{\epsilon_{i}= \pm 1}\left\|\sum_{i=1}^{n} \epsilon_{i}\right\| f_{i}\left\|\varphi_{i}\right\|
$$

$$
\|f\|\|\varphi\| \leqq \max _{\epsilon_{i}= \pm 1}\left\|\sum_{i=1}^{n} \epsilon_{i}\right\| f_{i}\left\|\varphi_{i}\right\|
$$


This proves

$$
N(f \otimes \varphi)=\|f\|\|\varphi\| \text {. }
$$

Definition 7.2. We define a crossnorm $N$ in $\mathfrak{A}\left(E_{1}, E_{2}\right)$ by means of the least crossnorm $N_{0}$ in $\mathfrak{A}\left(\bar{E}_{1}, \bar{E}_{2}\right)$. Let $k$ denote a natural number. For $\sum_{i=1}^{n} f_{i} \otimes \varphi_{i}$ in $\mathfrak{A}\left(E_{1}, E_{2}\right)$ we put

$$
\begin{aligned}
N_{(k)}\left(\sum_{i=1}^{n} f_{i} \otimes \varphi_{i}\right) & \\
& =\sup \left(\left|\left(\sum_{j=1}^{k} F_{j} \otimes \phi_{j}\right)\left(\sum_{i=1}^{n} f_{i} \otimes \varphi_{i}\right)\right| / N_{0}\left(\sum_{j=1}^{k} F_{j} \otimes \phi_{j}\right)\right)
\end{aligned}
$$

where sup (the least upper bound) is taken for all sequences of $k$ terms $F_{1}, \cdots, F_{k}$ in $\bar{E}_{1} ; \phi_{1}, \cdots, \phi_{k}$ in $\bar{E}_{2}$.

THEOREM 7.2. For every natural $k, N_{(k)}$ is a crossnorm.

Proof. I. If $\sum_{i=1}^{n} f_{i} \otimes \varphi_{i} \simeq 0 \otimes 0$, Lemma 2.1 gives $N_{(k)}\left(\sum_{i=1}^{n} f_{i} \otimes \varphi_{i}\right)=0$. If $N_{(k)}\left(\sum_{i=1}^{n} f_{i} \otimes \varphi_{i}\right)=0$, then taking $F_{1}=\cdots=F_{k}=F ; \phi_{1}=\cdots=\phi_{k}=\phi$, we have $\sum_{i=1}^{n} f_{i} \otimes \varphi_{i} \simeq 0 \otimes 0$ by Definition 4.2 and Lemma 4.4 .

That II, III, and IV hold is obvious. We shall prove that $N_{(k)}$ has the cross-property. We have

$$
\begin{aligned}
& N_{(k)}\left(f^{0} \otimes \varphi^{0}\right)=\sup \left|\left(\sum_{j=1}^{k} F_{j} \otimes \phi_{j}\right)\left(f^{0} \otimes \varphi^{0}\right)\right| / N_{0}\left(\sum_{j=1}^{k} F_{j} \otimes \phi_{j}\right) \\
& \leqq \sup \left\{\left|\left(\sum_{j=1}^{k} F_{j} \otimes \phi_{j}\right)\left(f^{0} \otimes \varphi^{0}\right)\right|\left(\left\|f^{0}\right\|\left\|\varphi^{0}\right\| /\left|\left(\sum_{j=1}^{k} F_{j} \otimes \phi_{j}\right)\left(f^{0} \otimes \varphi^{0}\right)\right|\right)\right\} \\
&=\left\|f^{0}\right\|\left\|\varphi^{0}\right\| .
\end{aligned}
$$

But on the other hand, putting $F_{1}=\cdots=F_{k}=F ; \phi_{1}=\ldots=\phi_{k}=\phi$ we obviously get $N_{(k)}\left(f^{0} \otimes \varphi^{0}\right) \geqq\left\|f^{0}\right\|\left\|\phi^{0}\right\|$. This completes the proof.

\section{REFERENCES}

1. S. Banach, Théorie des opérations linéaires, Warsaw, 1932.

2. D. G. Bourgin, Closure of products of functions, Bull. Amer. Math. Soc. vol. 46 (1940) pp. $807-815$. 396-414.

3. J. A. Clarkson, Uniformly convex spaces, Trans. Amer. Math. Soc. vol. 40 (1936) pp.

4. M. M. Day, Reflexive Banach spaces not isomorphic to uniformly convex spaces, Bull. Amer. Math. Soc. vol. 47 (1941) pp. 313-317.

5. F. L. Hitchcock, The expression of a tensor or polyadic as a sum of products, Journal of Mathematics and Physics vol. 6 (1937) pp. 164-189.

6. - Multiple invariants and generalized rank of a p-way matrix or tensor, ibid. vol. 7 (1927) pp. 40-79.

7. D. Milman, On some criteria for the regularity of spaces of type (B). C. R. (Doklady) Acad. Sci. URSS. vol. 20 (1938) pp. 243-246. 
8. F. J. Murray, On complementary manifolds and projections in spaces $L_{p}$ and $l_{p}$, Trans. Amer. Math. Soc. vol. 41 (1937) pp. 138-152.

9. - Bilinear transformations in Hilbert spaces, ibid. vol. 45 (1939) pp. 474-507.

10. F. J. Murray and J. von Neumann, On rings of operators, Ann. of Math. (2) vol. 37 (1936) pp. 116-229.

11. J. von Neumann, On infinite direct products, Compositio Math. vol 6 (1938) pp. 1-77. 111-115.

12. - On certain topology for rings of operators, Ann. of Math. (2) vol. 37 (1936) pp.

13. R. Oldenburger Composition and rank of $n$-way matrices and multilinear forms, ibid vol. 35 (1934) pp. 622-657.

14. - Nonsingular multilinear forms and certain p-way matrix factorizations, Trans. Amer. Math. Soc. vol. 39 (1936) pp. 422-455.

15. B. J. Pettis, Proof that every uniformly convex space is reflexive, Duke Math. J. vol. 5 (1939) pp. 249-253.

16. H. Weyl, The theory of groups and quantum-mechanics, translated from German by H. P. Robertson, New York, 1931.

COLUMBIA UNIVERSITY, NEW YORK, N. Y. 